\title{
References
}

1) Anon. (1938). Lancet, I, 94.

2) Aubrey, J. (1813). "Letters written by Eminent Persons in the Seventeenth and Eighteenth Centuries: to which are added, Hearne's Journeys to Reading, and to Whaddon Hall, the seat of Browne Willis, Esq. and Lives of Eminent Men." London: Printed for Longman, Hurst, Rees, Orme, and Brown, Paternoster-Rowg; and Munday and Slatter, Oxford.

3) Boyle, R. (1688). "A Disquisition about the Final Causes of Natural Things", 157-158.-London: Printed by H.C. for John Taylor, at the Ship in St. Paul's Church-Yard.

4) Franklin, K. J. (1933). Facsimile edition, with introduction and translation, of "De venarum ostiolis", 1603, of Hieronymus Fabricius of Aquapendente.-Springfield, III., and Baltimore Md.: Charles C Thomas.

5) Gordon, M. H. (1947). Unpublished notes on a visit with Lord Horder to Coombe Lodge.

6) Hervey, Mary S. F. (1921). "The life, correspondence and collections of T. Howard, Earl of Arundel."-Cambridge: At the University Press.

7) Paget, C. G. (1929). "By-Ways in the History of Croydon."-Croydon: The Central Library, Town Hall.

8) Paget, C. G. (1937). "Croydon Homes of the Past."-Croydon: The Central Library, Town Hall.

9) Power, D'Arcy, (1917). “A revised chapter in the life of Dr. William Harvey, 1636." Proc. R. Soc. Med., 10, 33-59. Reprinted in:

10) Power, D'Arcy, (1931). "Selected writings 1877-1930."-Oxford: At the Clarendon Press.

11) Spencer, H. R. (1921). Harveian Oration.

\section{Uber Lichtenbergs Anteil an der Ophthalmologie seiner Zeit}

\section{Von F.P. Fischer, Utrecht}

Der Zufall spielte mir einen Brief von Georg Christoph Lichtenberg an Goethe zu, der zwar in Lichtenbergs Briefen, herausgegeben von Leitzmann und Schüddekopf, Leipzig 1901, aufgenommen ist und auch schon vorher im Goethe-Jahrbuch, 18. Band, 1897, abgedruckt wurde, den Augenärzten aber ebenso unbekannt zu sein scheint, wie er mir war. Ich will diesen Brief, der der wichtigste ist aus dem Briefwechsel Lichtenbergs mit Goethe über das Farbensehen, darum hier wiedergeben. Wie sicherlich jeden, der diesen Brief liest, veranlaßte er auch mich, in Lichtenbergs Schriften nach weiteren Spuren seiner Beschäftigung mit den 
Problemen des Sehens und der Optik zu suchen. Der Brief zeigt eine für die damalige Zeit ganz ungewöhnliche Vertrautheit mit den Angelegenheiten der physiologischen Optik und verrät einen so meisterhaften Beobachter, daß man neugierig wird, den weltweiten Geist, der Lichtenberg war, im kleinen am Werk zu sehen.

Lichtenberg, geb. am 1. Juli 1742 in Oberramstädt bei Darmstadt, gest. am 24. Juli 1799 in Göttingen, im Leben königlich-großbritannischer Hofrat und Professor der Physik in Göttingen, klein, verwachsen, die letzten fünfzehn Jahre seines Lebens in sein Arbeitszimmer vergraben, ein menschenscheuer Menschenfreund, hat keine großen Werke hinterlassen, aber die Spuren seines Einflusses und sein Wirken sind heute noch lebendig. Freilich nicht in der Physik und Mathematik. Goethe sagte von ihm: "Lichtenbergs Schriften können wir uns als der wunderbarsten Wünschelrute bedienen; wo er einen Spaß macht, liegt ein Problem verborgen!»1 Seine ersten Herausgeber schilderten ihn so: «Es ist fast kein Zweig der menschlichen Wissenschaften, auf den Lichtenberg nicht einen neuen, fruchtbaren Keim gepfropft, - kein geheimer Winkel des menschlichen Kopfes und Herzens, wohin er nicht sein Diogenes-Lämpchen gehalten hätte, ein geistiger Midas, verwandelte er alle, auch die unbedeutendsten Gegenstände, die sein Wert berührt, in Gold, - ein neuer Prometheus, alle, auch die trockensten und abstraktesten, in Blut und Leben.» Lichtenberg war ein Meister des Wortes, und seine Aphorismen, die heute wohl mehr verwendet als gelesen werden, enthalten Formulierungen von Ewigkeitswert. "Lichtenberg gräbt tiefer als irgendwer, aber er kommt nicht wieder herauf. Er redet unter der Erde.

1 Nicht immer war Goethe auf Lichtenberg so gut zu sprechen. Während er seiner 1791 in den Beiträgen zur Optik z. B. $\$ 28$ ehrenvoll als Herr Hofrat Lichtenberg erwähnt, der für färbende Körper die Bezeichnung Pigmente in der 5. Auflage von Erxlebens Naturlehre (S.315) eingeführt habe, schreibt er in den Tag- und Jahresheften unter 1795: «Lichtenbergs Hogarth und das Interesse daran war eigentlich ein gemachtes: denn wie hätte der Deutsche, in dessen einfachen reinen Zustande sehr selten solche exzentrische Fratzen vorkommen, hieran sich wahrhaft vergnügen können? Nur die Tradition, die einen von seiner Nation hochgefeierten Namen auch auf dem Kontinent hatte geltend gemacht, nur die Seltenheit, seine wunderliche Darstellungen vollständig zu besitzen, und die Bequemlichkeit, zu Betrachtung und Bewunderung seiner Werke weder Kunstkenntnis noch höheren Sinnes zu bedürfen, sondern allein bösen Willen und Verachtung der Menschheit mitbringen zu können, erleichterte die Verbreitung ganz besonders, vorzüglich aber, daß Hogarths Witz auch Lichtenbergs Witzeleien den Weg gebahnt hatte.» 
Nur wer selbst tief gräbt, hört ihn» (Karl Kraus, «Sprüche und Widersprüche", Wien 1924). Als Mann der Wissenschaft gehört er der Geschichte, aber selbst da gilt noch sein Wort, damals wie heute: «Das Wissen aus einem Buch ins andere geht am Kopf vorbei.»

Der folgende Brief Lichtenbergs ist die Antwort auf drei vorhergegangene Brief Goethes, deren erster 1792 abgesandt wurde. Den dritten schickte Goethe zugleich mit einem Paket, das wahrscheinlich das Manuskript "Von den farbigen Schatten» enthielt, im gleichen Jahr entstanden, am 11. August 1793 von Frankfurt an Lichtenberg, der ihn am 21. September 1793, wie er ausdrücklich in seinem Tagebuch vermerkte, las und am 7. Oktober 1793 beantwortet hat. Aus dem Briefwechsel (Goethes Briefe X, 132, 145) mit Herder und Knebel geht hervor, wie viel Goethe an Lichtenbergs Meinung gelegen war, und wie sehr er wünschte, «daß dieser Mann meiner Untersuchung Freund bleibe.» Auch in der Farbenlehre erweist Goethe Lichtenberg mehrfach seine Reverenz, so im $\S 584$, didaktischer Teil, und in der "Confession des Verfassers». Nun der Brief Lichtenbergs:

«Alles, was ich seit einigen Jahren unternehme, geht sehr langsam vonstatten und, was das Betrübendeste bei der Sache ist, obendrein meistens ohne den Vorteil, den ein altes Sprichwort sonst einem solchen procedere zusichert, ich meine ohne den einer besseren Ausführung. Ich leide noch immer außerordentlich an Nerven, und es wird nun auch wohl nicht besser werden, bis ich die Nerven selbst ablege. ${ }^{2}$ Ich rechne daher sehr auf Ew. Hochwohlgeborenen Exzellenz geneigte Gesinnungen gegen mich, bei dieser Verzögerung meiner Antwort.

Ew. Exzellenz hätten mir nicht leicht ein größeres Vergnügen machen können als durch die gütige Mitteilung Ihres vortrefflichen Aufsatzes. Sie haben mich dadurch auf einen Teil der Lehre vom Lichte aufmerksam gemacht, um den ich mich bisher wenig gekümmert hatte. Es waren mir zwar einige der gemeinsten Phänomene bei den farbigen Schatten bekannt, aber, die Wahrheit zu gestehen, ich hatte nicht gedacht, daß dort noch so vieles läge, das einer weiteren Entwicklung so sehr bedürfte.

2 Lichtenberg soll im Alter von acht Jahren bei einem Fall die Wirbelsäule verrenkt haben. «Er war in seinen letzten Jahren, wahrscheinlich durch die immer mehr zunehmende Entstellung seines Körpers im eigentlichen Verstande menschenscheu so, daß er nicht nur oft Jahre lang sein Zimmer nicht verließ, sondern auch niemanden, außer höchstens ein paar sehr vertraute Freunde bei sich sehen wollte.» (L. Ch. Lichtenberg [der Sohn] und Fr. Kries.) 
Die Sache ist sehr angenehm und soll mich, wenn es meine Kräfte verstatten, nicht wenig beschäftigen.

Ehe ich Ew. Hochwohlgeboren einige meiner Gedanken über die Sache vortrage, $\mathrm{mu} ß$ ich dieselben vor allen Dingen auf eine Schrift über diese Materie aufmerksam machen, die Dr. Gehler in seinem physikalischen Wörterbuch ${ }^{3}$, Artikel: Schatten (blaue), Band III, Seite 826, anführt, weil sie nicht allein eine große Menge von zum Teil sehr schönen Versuchen enthält (92 an der Zahl), sondern weil der scharfsinnige Verfasser am Ende auf eine Erklärung des Phänomens gerät, die mit der Ihrigen auf eines hinausläuft. Der vollständige Titel ist: ,Observations sur les ombres colorées, contenant une suite d'expérences sur les différentes couleurs des ombres, sur les moyens de rendre les ombres colorées, et sur les causes de la différence de leur couleur. Par H. F. T. à Paris 1782 in groß 12 ms.' Daß dieses Mannes Theorie von der von Ew. Hochwohlgeboren nicht sehr verschieden ist, werden Sie schon aus dem wenigen, was Dr. Gehler davon sagt (vermutlich nach Brisson), ersehen können. Da ich das Buch selbst in Händen habe, so setze ich noch ein kurzes Resultat aus seinem Versuche mit des Verfassers eigenen Worten her: ,Tout ceci prouve bien', sagt er pag. 197, ,q'une certaine proportion de clarté entre les lumieres est non seulement nécessaire pour colorer les ombres, mais encore que leurs différentes couleurs dépendent aussi de la proportion d'intensité entre les mêmes lumieres.' Ich wünsche sehr, daß

3 «Physikalisches Wörterbuch oder Versuch einer Erklärung der vornehmsten Begriffe und Kunstwörter der Naturlehre mit kurzen Nachrichten von der Geschichte der Erfindungen und Beschreibungen der Werkzeuge begleitet in alphabetischer Ordnung von Dr. Johann Samuel Traugott Gehler im Schwickertschen Verlage Leipzig.» Schon 1798 in neuer Auflage erschienen. Zählt im Artikel «Schatten» fast alle Vorgänger Goethes auf und gibt als Quellen neben Priestleys «Geschichte der Optik» auch Brisson, Dict. rais. de Physique, Art. «Ombre» an. Durch Brisson kam Gehler zur Arbeit von H.F.T. über den heute ebensowenig bekanntgeworden ist als zu Goethes Zeiten. Der "Gehler» war seinerzeit ein sehr weitverbreitetes Buch, und sowohl Purkinje in den «Beiträgen zur Kenntnis des Sehens in subjektiver Hinsicht» als auch Joh. Müller in der "Vergleichenden Physiologie des Gesichtssinnes» erwähnen ihn öfters, ja selbst Helmholtz hat sich dieses Buches nachweisbar noch bedient. Goethe hat Fischers «Physikalisches Wörterbuch» bevorzugt, was besagen soll: zu Rate gezogen; er fand es langweilig und schlecht, immerhin weiß man von ihm selbst, daß er einige Teile dieses Buches bei sich hatte, als er 1792 bei Longwy und Valmy stand, wie in den Tag- und Jahresheften zu lesen ist. Nichts domonstriert wohl sein Interesse für optische Fragen besser als sein Studium im Jahre 1792 und 1793, wo er die Belagerung von Mainz mitmachte und dabei unablässig an die Farbenlehre dachte. 
Ew. Exzellenz einmal dieses Buch sehen könnten, und erbiete mich daher, falls es, wie ich fast vermute, in Ihrer Gegend nicht anzutreffen sein sollte, Ihnen das Exemplar von hiesiger Bibliothek zu übersenden, wenn Sie es befehlen. Es scheint das Aufsehen nicht gemacht zu haben, das es zu machen verdient. Der Verfasser hat sehr viel nette Versuche: unter anderem hat er mittels zweier Käfige, deren Schatten einander kreuzten, und bei dem Lichte von zwei Talglichtern und einem Kaminfeuer rote, violette, gelbe, blaue und grüne Schatten zu gleicher Zeit gesehen. Wenn anders die Beobachtungen nicht durch die Phantasie zu des Verfassers Zweck etwas abgerundet worden sind, welches gerade in diesem Teil der Physik, bei voller Unschuld des Beobachters, leichter als in irgendeiner anderen geschehen kann, wovon ich am Ende noch etwas sagen werde. Trotz der frappanten Versuche, womit Ew. Hochwohlgeboren Ihre Theorie unterstützen, und so sehr derselben auch die Beobachtungen des ebengenannten Herrn T. zustatten zu kommen scheinen, so kann ich mich doch, nach einigem, was ich beobachtet habe, noch nicht entschließen, sie für ganz ohne Einschränkung richtig zu erkennen. Ich rechne nämlich bei der ganzen Schattengeschichte sehr viel auf die Unbestimmtheit der Ausdrücke weiß, weißes Papier, usw. Die Menschen wissen freilich, was das für eine Farbe ist, die sie weiß nennen, aber wie vielen mag wohl je die reine weiße Farbe zu Gesicht gekommen sein? Im gemeinen Leben nennen wir weiß nicht, was weiß aussieht, sondern was weiß aussehen würde, wenn es dem reinen Sonnenlichte ausgesetzt wird oder doch einem Lichte, das der Qualität nach nicht sehr von dem Sonnenlichte abweicht. Es ist mehr die Disposition zum Weißwerden und Weißseinkönnen, in allen ihren Gradationen, was wir an den Körpern weiß nennen, als ihre reine weiße Farbe selbst. Ich halte diesen Bogen Papier z. B. für weiß, in der tiefsten Dämmerung, selbst in der Nacht beim schwächsten Sternenlicht, bei Talg-, Wachs- und Lampenlicht, im höchsten Sonnenschein, in der Abendröte, bei Schnee- und Regenwetter, im Walde und im tapezierten Zimmer usw., ich bin aber überzeugt, daß er, den höchsten Sonnenschein, etwa auf einer Alpenspitze ausgenommen, wo man noch den Widerschein des blauen Himmels entfernt hätte, nichts weniger als weiß ist. Wir merken dies freilich nicht, weil in allen unseren Urteilen, die sich auf Gesichtsempfindungen gründen, Urteil und Empfindung so zusammenwachsen, daß es uns in gewissen Jahren kaum möglich ist, sie wieder zu trennen, wir glauben jeden Augenblick etwas zu empfinden, was wir eigentlich bloß schließen. Da- 
her rührt es, daß die schlechten Porträtmaler die Gesichter ganz über und über mit Fleischfarbe anstreichen, sie können sich gar nicht vorstellen, daß in einem Menschengesicht blaue, grüne, gelbe und braune Schatten sein können, und bei ihrem Manschettenwerk verfahren sie so säuberlich, daß man nur aus dem Ort und dem Umriß errät, daß der Kalkfleck, den sie hingekleckst haben, eine Manschette vorstellen soll. Meinem Fenster gegenüber steht ein weißer Schornstein, dessen beide mir sichtbaren Seiten selten einerlei Grad von Erleuchtung haben. Zuweilen, wenn mir die eine Seite gelb oder bläulich zu sein scheint, frage ich Personen von übrigens sehr richtigem Verstande um die Farben des Schornsteins. Gewöhnlich ist die Antwort, er ist so weiß auf der einen Seite als auf der anderen, auf die eine scheint aber die Sonne, das macht den Unterschied. In der Camera obscura fallen die Urteile schon richtiger aus, daher wird auch das Kolorit leichter nach den Werken großer Meister als nach der Natur studiert, weil man dort die Farbe schon vom Urteil geschieden auf der Leinwand hat, und sie wie jeden anderen gefärbten Lappen untersuchen kann gegen allerlei Licht und in allerlei Neigungswinkeln gegen dasselbe, hier aber erst Urteil von Empfindung geschieden werden muß, das nicht jedermanns Sache ist. Mit einem Worte: Weiß nennen wir die Disposition der Oberfläche eines Körpers, alle Arten gefärbten Lichts gleich stark nach allen Richtungen zurückzuwerfen, und ein solcher Körper erscheint auch wirklich weiß, wenn jenes gefärbte Licht, der Menge sowohl als der Beschaffenheit und Intension nach, auf ihn fällt, in allen anderen Fällen nicht. Es läßt sich fast das Unendliche gegen eins verwetten, daß ein Körper, z. B. ein Bogen weißes Papier, der die Kapazität zur Weiße im höchsten Grade besitzt, nie eigentlich weiß erscheinen werde und nie auf einem Gemälde weiß dargestellt werden dürfte. Z. B. ich schreibe jetzt einem Fenster gegenüber, das nach Mitternacht sieht, der Himmel ist ziemlich heiter, und mehrere Dächer, die gegen Mittag und Abend gerichtet sind, werden von der Sonne etwas beschienen, mein Zimmer ist himmelblau tapeziert, die weiße Decke desselben wird beträchtlich durch die gegenüberstehenden Häuser erleuchtet, was für mannigfaltiges Licht fällt nicht auf dieses Blatt? Daß aber alle die Farben dieser Gegenstände auf dem Papier liegen, bedarf, dünkt mich, keines Beweises. Denn wenn ich das Zimmer ganz verfinstern und nun nach Belieben bald hier und da ein Loch in die Wand stechen könnte, so würde sich auf ihm allemal die Farbe eines Gegenstandes an der Stelle zeigen, die mit dem Gegenstand und dem Loch in einer ge- 
raden Linie läge. So wie ich also, nachdem ich das Loch bald hier, bald da bohrte, blaue, rote und gelbe usw. Flecke auf meinem Papier hervorbringen könnte, so entstehen auch die Schatten auf einem sogenannten weißen Papier, wenn Licht abgehalten wird, das nötig ist, die sogenannte Weiße (die es aber nicht ist) hervorzubringen. Daß der blaue Schatten nicht vom blauen Himmel herkomme, haben Ew. Hochwohlgeboren, glaube ich, unwiderleglich dargetan, allein ich glaube, daß auch beim trüben Himmel entweder das Blaue immer herrschend sei, oder daß wenigstens das Graue in der Nachbarschaft von dem Gelben uns blau erscheine. Dieses habe ich auf eine Weise erfahren, die mir keinen Zweifel übrig läßt. Ich besitze einen Planspiegel von fast orangegelbem Glase. Mit dem setzte ich mich an die vom Fenster am weitesten entfernte Wand. Es war ein sehr trüber Tag. Als ich in diesem Spiegel das Fenster mit seinen dunklen Stäben betrachtet, so sah ich manche darunter dreimal, einmal schwarz, einmal orangegelb und einmal himmelblau. Ich erklärte mir die Erscheinung so, und ich glaube nicht, daß ich mich irre. Der Spiegel machte zwei Bilder, eines von der Oberfläche wie jede andere Spiegelscheibe und eines von der Belegung, das Bild eines Stabes von der Belegung her war schwarz, wenn es zugleich mit dem Bild eines Stabes von der Oberfläche her zusammentraf, orangefarb, wenn das Bild von der Oberfläche her den hellen Himmel von der Belegung her unter sich hatte, und himmelblau, wenn das Bild von der Belegung her den hellen Himmel von der Oberfläche her über sich hatte. Es sah vortrefflich aus. Sobald ich aber eine gelbe, nicht belegte Spiegelscheibe daneben legte, so fand ich aus der Vergleichung, daß das, was ich in schmalen Streifen zwischen den vielen und herrlichen gelben für Himmelblau hielt, weiter nichts war als das Graue des Himmels, das ich auf der Spiegelscheibe sah, und endlich fing ich sogar an, meine himmelblauen Streifen nicht mehr für blau zu halten. Ob ich mir gleich einiges in Ew. Exzellenz Versuchen noch nicht erklären kann, so möchte doch dieses vielleicht geschehen, wenn mir das Lokale, auf welches hier außerordentlich viel ankommt, ganz bekannt wäre. Auch dieses habe ich erfahren. Vor etwa zehn Tagen, da die Sonne auf meinen Gang um zwölf Uhr schien, hielt ich einen Schlüssel gegen eine weiße Wand, die durch bloße Reflexion erleuchtet war, und fand den Schatten blaß lila. Heute um zwölf Uhr, da die Umstände bis etwa die verschiedene Deklination der Sonne den vorigen sensibiliter gleich waren, hielt ich den Schlüssel an dieselbe Wand, und der Schatten war schmutzig gelblich. Haben Ew. Hochwohl- 
geboren wohl auch schon die herrlichen lila Schatten gesehen? Da ich seit dem Empfang Ihres Schreibens den bunten Schatten nachlaufe wie ehemals als Knabe den Schmetterlingen, so hatte ich neulich in meiner Kammer unvermutet einen herrlichen Anblick. Es herrschte in dieser Kammer, worin ich Bücher stehen habe, ein sonderbares, ungewisses, magisches Licht, dem man ansah, daß es das Produkt durcheinander geworfener Bilder von gegenüber befindlichen und von der Sonne beschienenen Gegenständen war, denen ein halb herabgelassener weißer Vorhang den Eingang zum Teil erschwerte. Gleich stellte ich am entferntesten Ende vom Fenster einen Bogen Papier auf, als ich meine Hand dagegen hielt, war der Schatten lila und nah angehalten schwarz mit lila Einfassung, und zur Seite lagen zwei bis drei blaßgrüne Schatten. Ein dicker Bleistift, horizontal gehalten, zeigte nur einen lila Schatten, vertikal, lila und blaßgrüne nebeneinander.

Ohne mich weiter in meine Erklärung einlassen zu dürfen, werden Ew. Exzellenz schon sehen, wo ich hinaus will, ich lasse also die Anwendung weg. Doch will ich damit gar nicht sagen, daß nicht irgend hierein etwas noch zurück sei, das anders erklärt werden muß. Es ist z. B. gewiß, daß, wenn man lange durch ein rotes Glas sieht und zieht es plötzlich vor den Augen weg, so erscheinen die Gegenstände einen Augenblick grünlich, sieht man hingegen durch ein grünes Glas, so erscheinen sie alsdann anfangs rötlich. Dieses hängt mit Buffons couleurs accidentelles ${ }^{4}$ zusammen, die man in den Augen bemerkt.

Mit einem Wort, ich glaube, die Sache ist sehr wichtig, und ich verspreche mir von Ew. Exzellenz Bemühungen nach diesem herrlichen Anfange sehr viel. Ich werde gewiß, soviel es die Umstände verstatten, mitarbeiten und nicht versäumen, denselben Nachricht zu geben.»

Schon die Einleitung dieses Briefes mit dem Hinweis auf die bereits bestehende Literatur läßt ihn als ein Kabinettstück didaktischen Stils erscheinen. Man bedenke, daß es auch in dem viel briefwechselnden 18. Jahrhundert doch ein seltenes Vorkommnis war, daß ein Mann von der gesellschaftlichen Stellung Lichtenbergs, der schließlich nur Professor an einer ausländischen Universität war, was damals wohl kaum etwas vorstellte, sich so freimütig zu einem in seinem Land allmächtigen und in der übrigen Welt damals schon als Dichter, Gelehrter und Minister

4 Buffon, Mém. de l'Acad. de Paris, p. 217, 1743. 
überaus geschätzten hohen Herrn äußerte. Wiewohl der Brief in jeder Hinsicht die persönliche Wertschätzung verrät und jeder Etikette taktvoll Rechnung trägt, ist es doch durchaus ein Brief, wie ihn eben ein erfahrener und einsichtiger Fachmann an einen im Fache noch nicht so betriebenen schreibt, dem er helfen will, den rechten Weg zu finden. Daher folgen der Einleitung sogleich sachliche Bemerkungen, die sich wieder ganz zwanglos zu Besprechungen von Versuchen verwenden lassen, wobei hie und da kritische Bemerkungen eingeschoben werden können, etwa daß farbige Schatten keineswegs blau sein müssen, wohl aber am leichtesten im Sonnenlicht bei blauem Himmel zu erzeugen seien. So ergibt sich spielend das wunderschöne Experiment mit dem orangegelben Spiegel, mit dem Schlüssel und im magisch beleuchteten Bücherkämmerchen, wo Gelegenheit zur Beobachtung des Simultankontrastes war, was mit einem Hinweis auf den Sukzessivkontrast, wie wir heute sagen, wieder bei der schon bekannten Literatur anschließt.

Goethe antwortete noch im selben Monat, doch ist nur ein Konzept nach einem Diktat erhalten geblieben, geschrieben von der gleichen Hand, die auf den Faszikel, der im Goethe- und Schiller-Archiv bewahrt ist, "Älteste Papiere zur Farbenlehre» schrieb. In diesem Faszikel lag auch der Brief Lichtenbergs. Aus dem Verzeichnis der Postsendungen in Weimar ist zu ersehen, daß der Brief an Lichtenberg am 23. Oktober 1793 abging. Er findet sich in der Sophien-Ausgabe von Goethes Werken in der IV. Abteilung, 10. Band, Seite 116, und trägt die Nummer 3021. Ich will ihn nur auszugsweise wiedergeben.

«Zuerst danke ich für die Bekanntschaft, die Sie mir mit der französischen Schrift verschaffen, ich bitte mir solche möglich zu überschicken, denn wir mögen noch so geneigt sein, auf Zweifel und Widerspruch zu hören, so ist es doch unserer Natur gar zu gemäß, dasjenige begierig zu ergreifen, was mit unserer Vorstellungsart übereinkommt.

Nach diesem aufrichtigen Bekenntnis bitte ich Ew. Wohlgeboren, mich eben für so aufrichtig zu halten, wenn ich versichere, daß Ihre Bedenklichkeiten mir von dem größten Gewichte sind. Können Sie sich manches in meinem Aufsatze nicht ganz erklären, scheint Ihnen die Reihe der Experimente nicht so rein, die daraus gezogenen Folgerungen nicht so überzeugend, so muß mich das auf meine Versuche, auf meine Methode und mein Urteil mißtrauisch machen. Ich werde meinen französischen Kollegen sorgfältig studieren, sowohl seine Versuche als das, 
was mir bisher Neues bekannt geworden, nachtragen, auf Ew. Wohlgeboren Bemerkungen alle Rücksicht nehmen und die Resultate meiner Arbeit abermals vorlegen.»

Nach dieser Einleitung, die wohl jeder brave und eifrige Schüler geschrieben haben könnte, folgt, was Goethe «eben zuletzt dringender ward und das eckelhafte Newtonsche Weiß mit Gewalt verfolgte» nannte (siehe Seite 84). Denn niemals wieder ist in dem Briefwechsel mit Lichtenberg von Weiß die Rede.

«Was Ew. Wohlgeboren über das Weiß in Ihrem Briefe äußern, scheint mir der Lehre gemäß zu sein, welche das Weiß aus vereinigten Farben entstehen läßt. Ich behalte mir vor, meine Vorstellung hierüber vorzulegen und Threr Prüfung zu unterwerfen.»

Das ist zwar richtig, aber wenig. Doch sogleich verfolgt Goethe mit neuen Experimenten, anknüpfend an den orangefarbenen Planspiegel, die ihn als den Meister treuer Beobachtung zeigen, der er immer war.

«Das Phänomen, das Ew. Wohlgeboren in dem orangefarbenen Planspiegel bemerkt, habe ich unter die Zahl derjenigen aufgenommen ${ }^{5}$, welche uns die Reflexion darstellt. Ist der Spiegel blau, so erscheint das Phänomen umgekehrt, das von der Oberfläche zurückgeworfene Bild des Stabes erscheint nunmehr blau, das von der Belegung gelb, gelbrot, bräunlich rot! Ist der Spiegel grün, so erscheint das obere Bild grün, das untere violett oder purpur, je der Zeit mit den entgegengesetzten Farben, wie bei den farbigen Schatten. Man nehme bei dem gewöhnlichen Versuche, wo man Kerzenlicht dem schwachen Tageslicht entgegensetzt, ein hellgrünes Glas und halte es vor das Licht, sogleich wird der gelbe Schatten grün, der blaue hingegen purpurroth oder violett erscheinen. Man kann diesen Versuch auch noch auf eine auffallende Weise vermännigfältigen: Man lege bei heiterem Himmel und hellem Sonnenschein ein weißes Papier ins Freie, man halte einen Stab darauf und der Schatten wird mehr oder weniger bläulich erscheinen. Man nehme darauf eine grüne Glasscheibe und lasse das Sonnenlicht durch selbige auf das Papier fallen, davon ein Teil also grün erscheinen wird, man stelle den Stab in dieses grüne Licht, und der Schatten desselben wird sogleich violett erscheinen. Ebenso ist der Schatten gelblich, wenn das Glas blau, blau, wenn das Glas gelb ist. Grau ist aber und bleibt der Schatten, auch

5 Der Farbenlehre didaktischer Teil, Physiologische Farben, p. 80. 
mitten im gefärbten Lichte, wenn man den Versuch am Fenster einer Camera obscura macht und die Einwirkung des Tageslichtes abhält.

Das Phänomen, dessen Sie gegen das Ende Ihres Briefes erwähnen, habe ich neulich in einem eminenten Grade gesehen. Ich betrachtete durch die öffnung der Camera obscura die Sonne durch ein dunkelviolettes Glas, deren Scheibe mir denn in dem lebhaftesten Purpur erschien, als ich wieder hereinsah und mein Blick auf einen schwarzen Mantel fiel, so erschien mir dieser vollkommen grün. Einige Zeit vorher war ich auf folgende Versuche geleitet. An eine weiße Wand stellte ich ein etwa dreizöllig viereckiges gelbes Papier und sah scharf darauf, sodann blickt ich in die Höhe und richtete meine Augen unverwandt auf einen bestimmten Fleck der weißen Wand: An gedachtem Platze erschien mir bald ein bläuliches Viereck, so wie im Gegenteil mir ein gelbes erschien, wenn das untere Viereck blau war, und so veränderte sich auch bei veränderten Farben des Gegenstandes die Farbe der Erscheinung nach den Gesetzen, wie sie mir aus den Phänomenen der farbigen Schatten zu folgen schienen. Auch hiervon will ich, was mir bekannt ist, zusammen schreiben und vorlegen, mit der Bitte, die Specimina eines Liebhabers und Autodidakten freundlich aufzunehmen.».

In einem späteren Brief vom 9. Juni 1794 findet sich noch die Stelle:

"Wenn es Ihre Zeit erlaubt, so haben Sie ja die Güte, mir mit Ihren Bemerkungen über meinen letzten Aufsatz zu helfen. Sein Sie nur versichert, daß ich jede Art von Rektifikation und Widerspruch vertragen kann.»

Lichtenberg aber hat nicht mehr geantwortet, der weitere Briefwechsel geht um Austausch von Veröffentlichungen und Rückgabe entliehener Bücher und schließlich bleiben die Briefe ganz aus. Bitter beklagt sich Goethe darüber in einem Briefe an Schiller $(10,335)$ und wohl das deutlichste Zeichen seiner Enttäuschung ist die bekannte Stelle aus der Konfession des Verfassers: "mit Lichtenberg korrespondierte ich eine Zeitlang und sendete ihm ein paar auf Gestellen bewegliche Schirme, woran die sämtlichen subjektiven Erscheinungen auf eine bequeme Weise dargestellt werden konnten, ingleichen einige Aufsätze, freilich noch roh und ungeschlacht genug. Eine Zeitlang antwortete er mir, als ich aber zuletzt dringender ward und das ekelhafte Newtonsche Weiß mit Gewalt verfolgte (siehe Seite 83), brach er ab, über diese Dinge zu schreiben und zu antworten, ja er hatte nicht einmal die Freundlichkeit, ungeachtet 
eines so guten Verhältnisses, meiner Beiträge in der letzten Ausgabe seines Erxlebens zu erwähnen.»

Lichtenberg hat den Erxleben fünfmal herausgegeben und Goethes Beiträge niemals erwähnt, sich freilich über das Farbensehen, das nach den Auffassungen von damals in den Lehrbüchern der Physik behandelt wurde, recht kurz gefaßt.

Herr Professor R. H. K a h $\mathrm{n}$ hat mich auf den folgenden Sachverhalt aufmerksam gemacht, der einiges Licht wirft auf die "Gewalt», mit der Goethe das "ekelhafte Newtonsche Weiß» verfolgte, was nach Goethe die Ursache war, daß Lichtenberg abbrach, «über diese Dinge zu schreiben und zu antworten». So nachhaltig muß dies auf Goethe gewirkt haben, daß er bis 1810, in welchem Jahre "Zur Farbenlehre» erschien, Lichtenberg, und was mit ihm zusammenhing, nicht vergessen konnte. Lichtenberg war 1799 gestorben. Zur Einführung will ich die beiden Paragraphen 583 und 584 des didaktischen Teils von "Zur Farbenlehre» wörtlich zitieren: «Jede Farbe also, um gesehen zu werden, muß ein Licht im Hinterhalte haben. Daher kommt es, daß je heller und glänzender die Unterlagen sind, desto schöner erscheinen die Farben. Zieht man Lackfarben auf einen metallisch glänzenden weißen Grund, wie unsere sogenannten Folien verfertigt werden, so zeigt sich die Herrlichkeit der Farbe bei diesem zurückwirkenden Licht so sehr als bei irgendeinem prismatischen Versuche. Ja die Energie der physischen Farben beruht hauptsächlich darauf, daß mit und hinter ihnen das Licht immerfort wirksam ist. Lichtenberg, der zwar seiner Zeit und Lage nach der hergebrachten Vorstellung folgen mußte, war doch ein zu guter Beobachter, und zu geistreich, als daß er das, was ihm vor Augen erschien, nicht hätte bemerken und nach seiner Weise erklären und zurechtlegen sollen. Er sagt in der Vorrede zu Delaval: ,Auch scheint es mir aus anderen Gründen ... wahrscheinlich, daß unser Organ, um eine Farbe zu empfinden, etwas von allen Licht (weißes) zugleich mit empfinden müsse'.»

Das ist wohl ein schlagender Beweis, daß Lichtenberg vor dem ekelhaften Newtonschen Weiß die Augen aufgegangen sind, und als ein solcher Beweis wird dieses Zitat von Goethe auch, wie sich sogleich zeigen wird, angeführt. Denn deutlich zeigt diese Stelle aus einer Vorrede, daß Lichtenberg nicht "etwas von allem Licht (weißes) zugleich mit e m p f a n ge n", schrieb und dachte, was er als Newtonianer doch hätte tun müssen. 
Nun beschreibt Goethe ausführlich die Arbeit von E. H. Delava ${ }^{6}{ }^{6}$ in den "Materialien zur Geschichte der Farbenlehre», und zwar die deutsche Übersetzung von $\mathrm{Cr}$ e 11, zu welcher Crell eine Vorrede geschrieben hatte, worin wiederum zwei kurze Aufsätze von Klü gel, dem bekannten Übersetzer von Priestleys "Geschichte der Optik», und von Lichtenberg aufgenommen waren. Aus Lichtenberg zitiert Goethe nun zwei merkwürdige, unserer Überzeugung günstige Stellen, wovon wir die eine schon (§ 584) früher angeführt. Dem Zitat geht eine kurze Würdigung Lichtenbergs voraus, die man nicht gern wird unzitiert lassen können. "Merkwürdig ist besonders in dem Lichtenbergschen Aufsatz, wie man der Newtonschen Lehre durch chemische Hilfstruppen in jener Zeit wieder beigestanden. Man hatte eine latente Wärme ausgemittelt, warum sollte es nicht auch ein latentes Licht geben? und warum sollten die, nach der Theorie, dem Lichte angehörigen farbigen Lichter nicht auch der Reihe nach Versteckens spielen, und wenn es dem gelben beliebte, hervorzugucken, warum sollten die übrigen nicht neckisch im Hinterhalte lauschen können?» Dann folgt das erste Zitat aus Lichtenbergs Aufsatz: "Ich bemerke hier im Vorbeigehen, daß vielleicht die Lehre von den Farben eben deswegen bisher so viele Schwierigkeiten hatte, weil alles auf e i n e m Wege, z. B. Brechung, erklärt werden sollte.» Daß dieser Ausspruch Goethes Überzeugung günstig sei, kann heute wohl nicht behauptet werden, denn er besagt ja nur, daß Newtons Weg schwierig sei, aber nicht, daß er falsch wäre. Und nun folgt das zweite Zitat, schon aus $\$ 584$ bekannt, aber nun in dieser Form: "Auch scheint es mir aus anderen Gründen wahrscheinlich, daß unser Organ, um eine Farbe zu e $m p f$ ind e n, etwas von allem Licht (weißes) zugleich e mpf a $\mathrm{gen}$ müsse.» Also empfangen! Das ist doch ein großer und wesentlicher Unterschied gegenüber $\S 584$, wo e mp finden steht. Was aber hat Lichtenberg wirklich gesagt? Was steht im Original?

Das Original ist anscheinend sehr selten geworden und war nur unter Schwierigkeiten zu beschaffen. Auf Seite XXV oben des Lichtenbergschen Aufsatzes steht: "Auch scheint es mir aus anderen Gründen, die ich hier nicht anführen kann, wahrscheinlich, daß unser Organ, um eine Farbe zu empfinden, etwas von allem Licht (weißes) zugleich mit empfangen müsse: geschieht dieses nicht, zumal wenn die benachbarten Gegenstände sehr

6 Versuch und Bemerkungen über die Ursache der dauerhaften Farben undurchsichtiger Körper. Übersetzt und herausgegeben von Crell. Berlin und Stettin 1788, p. 8. 
helle sind, so sieht man die einzelne Farbe gar nicht, oder sind erstere alle sehr dunkel, so hält das Auge die Farbe für Feuer (doch dieses im Vorbeigehen). - Nun dieses auf Herrn Delavals Versuche angewandt. Seine Flasche ließ von dem schwachen Licht der Körper in der Stube von allen Farben etwas durch, also wirklich weißes, traf dieses hinten weißes Papier an, so würde es bei hellem Wasser schwach weiß erschienen sein, so aber wurde das einfallende im Hinweg etwas zersetzt: war also das Wasser rot, so schickte das weiße Papier sein Weiß mit jenem Übergewicht des Roten zurück, da es alle Farben zurücksendet; war die hintere Wand schwarz, so wurde nichts, auch das Rote nicht zurückgeworfen, und daher erschien die Flasche schwarz.»

Das ist lange vor dem Briefwechsel mit Goethe von Lichtenberg niedergeschrieben. Unzweifelhaft geht aus diesem Zitat hervor, daß Lichtenberg nicht nur empfangen schrieb, sondern auch wirklich dachte und meinte. Vielleicht wurden zwischen beiden noch andere Briefe gewechselt, die uns nicht erhalten geblieben sind, jedenfalls aber war Lichtenberg von der Newtonschen Lehre nicht abzubringen, und wenn ihm Goethe wirklich mit Gewalt zugesetzt haben sollte, dann blieb ihm auch nichts anderes übrig als abzubrechen und nicht mehr zu antworten. Doch hat Lichtenberg sich immer wieder mit dieser Materie beschäftigt, ich möchte fast sagen, sie hielt ihn so fest, daß er von ihr gar nicht mehr loskommen konnte, nur hat er nichts der öffentlichkeit bekanntgegeben, sondern, was er dachte, in seine Tagebücher vergraben. Ich will, was ich finden konnte - die Originale standen mir nicht zur Verfügung - nun folgen lassen.

"Die gefärbten Schatten verdienen gewiß die größte Aufmerksamkeit des Naturforschers. Die meisten denken zu leichtsinnig davon. Man glaubt die Sache schon ganz erklärt zu haben, und ich bin geneigt zu glauben, daß man sie noch nicht ganz geklärt hat, ja, was noch mehr ist, es könnte sein, daß der gegenwärtige Zustand der Optik oder unsere Kenntnis vom Lichte gar nicht einmal hinreichte, sie zu erklären, daß also folglich eine gründliche Auseinandersetzung dieses schweren Problems ein wahrer Gewinn sein würde. Ein Hauptbuch darin ist das kleine französische Werk, dessen Verfasser sich bloß mit H.F.T. bezeichnet, und das Herr Dr. Gehler in seinem Wörterbuche, Artikel ,Schatten', aufführt. Es hat fast ganz Herrn von Goethes Idee. Ich glaube doch noch immer, daß vieles bei Auflösung des Problems von den gefärbten Schatten auf einer genaueren Erörterung dessen beruht, was wir Weiß 
nennen. Weiß, sagt man, ist derjenige Körper, der alle Farben zurückwirft. Diese Definition setzt nicht allein stillschweigend voraus, daß alle Farben da sein müssen, um sich reflektieren zu lassen, wo man etwas Weißes sieht, sondern daß auch alle diese Farben in dem gehörigen Verhältnisse, sowohl der Quantität als Qualität nach, da sein müssen. Wo ist aber in der Welt dieses zu erwarten als etwa beim reinsten Sonnenlichte auf dem höchsten Punkte des Erdbodens! Was ist also die Folge davon? Wir sehen selbst im Sonnenlichte nie einen weißen Körper, und noch weniger im Schatten oder bei bedecktem Himmel. Allein ob wir gleich kein reines Weiß bemerken können, so wissen wir doch gar wohl, was wir unter Weiß verstehen. Denn wir korrigieren unsere Empfindungen immer durch Schlüsse. Dieses lernen wir so früh und wird uns so zur Natur, daß wir endlich zu empfinden glauben, was eigentlich ein Schluß ist. Bei der Wäsche macht die Person, die sie trägt, die Art der Falten usw., daß ich sie selbst an einem trüben Tage, oder in der Abend- oder Morgenröte immer für sehr weiß halte, da sie es gewiß nicht ist. Es wird bloß geschlossen und so mit allen Farben.»

Lichtenberg läuft hier der Auffassung von Helmholtz artig voraus. Es konnte auch gar nicht anders sein, denn die Idee der Farbe als Empfindung, ausgelöst durch verschiedene Wellenlänge, die Newton rein und deutlich aussprach, ist trotzdem das Kind einer späteren Zeit. Um so merkwürdiger, wenn sie doch bei Lichtenberg immer wieder so, wie in dem Briefe an Goethe, anklingt.

"Wenn Goethe und der französische Verfasser über die Schatten recht hätten, so könnte der blaue Himmel bloß der durch das Tageslicht erleuchtete Schatten sein, den das Licht der anderen Gegenstände im Auge wirft.»

«Es verhält sich mit der weißen Farbe bei meiner Schattenlehre wie mit den Parallel-Linien: wenn wir auch Parallel-Linien zeichnen, so sehen wir sie nicht parallel, wenn sie nicht auf einer Kugelfläche gezeichnet sind, in deren Mittelpunkt sich das Auge befindet.»

«Hat das Phänomen von den blauen und gelben Schatten nicht vielleicht Verwandtschaft mit dem Geschmackswesen in den galvanischen Versuchen mit der Zunge? Man schmeckt erst das eine, wenn das andere da ist. Ich glaube, daß diese Bemerkung Aufmerksamkeit verdient."

Mischen wir nicht heute gerade so Richtiges und Falsches?

«Daß man alles grünlich sieht, wenn man lange durch ein rotes Glas gesehen, und umgekehrt, rötlich, wenn man lange durch ein grünes ge- 
sehen hat, ist ein merkwürdiger Umstand. Es scheint sehr für Eulern ${ }^{7}$ zu streiten.»

"Vielleicht ist gar die Empfindung des Sehens bloß eine Zersetzung des Lichtes oder eine Verbindung verschiedener Stoffe unseres Körpers mit diesem einfachen Körper.»

Wie unglaubhaft modern dieser Ausspruch wäre, stände nicht «dieser einfache Körper»!

«Die Sehnerven sind doch beständig beschäftigt. Wenn ich in der dunkelsten Nacht im Bette liege und noch überdies die Augen schließe, so sehe ich doch immer kein volles Schwarz, sondern immer etwas mit Grau meliert.»

Hier sehen wir Lichtenberg Hering das Eigengrau der Netzhaut vorwegnehmen; ein solcher Ausspruch erscheint mir wichtiger als dicke Abhandlungen, die durch zeitbedingte zufällige Umstände verursacht sind. Vor allem aber zeigen sie den echten Naturforscher, der immer und unausgesetzt beobachtet und, was er findet, treu wiedergibt.

"Wir sehen alles auf eine Ebene, auf eine Glasscheibe projiziert, das Bild selbst, wovon unsere Vorstellungen abhängen, ist auf eine Kugel gezeichnet, der Hinterwand des Auges.»

Von Dürers Erklärung der Perspektive ausgehend, wird hier in einen Satz das ganze Problem der Abbildung und ihrer Fehler umrissen und das in einer Zeit, in der der Begriff des Astigmatismus noch nicht konzipiert war oder gar die Folgen schief inzidierender Büschel gekannt oder erkannt waren.

«Die Erscheinung von den Lichtspießen, die Meister den Augenwimpern zuschrieb, und worüber $\mathrm{Hr}$. Vieth in seinen mathematischen Abhandelungen geschrieben hat, verdienten immer noch eine neue Untersuchung. Hr. Vieth erklärt sie aus kleinen Runzeln auf der KristallLinse, welches der Rezensent dieser Schrift in der neuen allg. Bibl. (3. Bd., S. 41) mit Recht nicht wahrscheinlich findet. Die Versuche müßten unstreitig noch mehr variiert werden.»

Besonders interessant ist, daß diese lose hingeworfene Tagesnotiz doch so genau ist, daß ich mit ihrer Hilfe ohne jede Mühe die zitierten Bücher aus der hiesigen Universitätsbibliothek bekam, als handelte es sich um laufende Literatur und nicht um 147 Jahre alte Bücher. Meister

\footnotetext{
7 Euler ( NNova theoria lucis colorum in opusculis varii argumenti», Berlin 1746, und Mém. de l'Acad. de Prusse, p. 271, 1752) glaubte, daß das Auge achromatisch sei.
} 
las zu Lebzeiten Lichtenbergs in Göttingen Perspektive und Optik, Goethe nennt ihn in der Geschichte zur Farbenlehre unter "Academie Göttingen». Doch hat nicht er, sondern Priestley die zitierte Erklärung gegeben und zwar in seiner berühmten "History and present state of discoveries relating to vision", London 1772. Das Büchlein von Vieth hat den Titel "Vermischte Aufsätze für Liebhaber mathematischer Wissenschaften von G.U.A.Vieth, öffentlichem Lehrer der Mathematik zu Dessau, Berlin 1792. Es enthält u. a. einen Aufsatz über die Strahlenkränze, der voll interessanter Einzelheiten ist und wohl wert einer genauen Wiedergabe, die natürlich in diesem Zusammenhang nicht gegeben werden kann. Übrigens sei als Kuriosum mitgeteilt, daß diese Arbeit nirgendwo zitiert ist, obwohl aus einer Stelle bei Helmholtz hervorzugehen scheint, daß sie ihm, vielleicht durch einen anderen Autor, bekannt war. Die "Neue allgemeine deutsche Bibliothek» war ein jährlich erscheinendes Buch, Verlagsort Kiel, eine Art allgemeines Zentralblatt, das in etwa 22 Hauptkapiteln alle Erscheinungen eines Jahres referierte.

Da man nun gezeigt hat, daß der Bau des Auges nicht auf Achromatismus zielt, so entsteht die Frage: ist nicht vielleicht ein anderer Zweck dadurch erreicht worden? Aber, läßt sich nun fragen, an was für Augen hat man Krümmungen gemessen, an toten oder lebendigen? An lebendigen gewiß nicht. Und gesetzt auch, es gehe mit dem Tode keine Veränderung vor, so ist doch immer die Frage: ist auch das Auge vollkommen gewesen?

Könnten nicht der humor vitreus und aqueus bloß Gefäße sein, die zur Absonderung der Substanz der Kristall-Linse dienten, sowie etwa die Leber zur Absonderung der Galle, da sie doch nun einmal nicht achromatisch sein sollen? Aber wie verhält es sich mit anderen Augen, z. B. des Igels, von denen man sagt, sie haben bloß eine Linse?

Da es ausgemacht ist, daß das Licht noch mehr Nutzen hat, als dem Auge zu leuchten, und folglich auch aus anderer Absicht zuweilen dem organischen Körper nützen kann: so ist es doch noch eine Frage, ob alles, was wir an den Insekten für Augen halten, es auch wirklich sind.

Sollte wohl das Auge nur allein zum Sehen taugen? oder nicht auch ein Verdauungswerkzeug für das Licht sein? Ich kann mir auch gar nicht vorstellen, daß das $\mathrm{Ohr}$ allein zum Hören und die Nase zum Riechen dienen sollte.

Der Vorschlag, den Segner zu archimedischen Brennspiegeln tut, ließe sich vielleicht beim Katzenauge nützen, nämlich Nachts immer dahin zu leuchten, wohin man sieht, gleichsam eine leuchtende Chorioidea vorzustellen. Sollte nämlich nicht ein Tubus möglich sein, der mit einem Erleuchtungsapparat verbunden, immer gerade dahin leuchtet, wohin man hinsehe. 
Segner war ebenfalls in Göttingen Professor der Mathematik und Medizin. Er hat eine Schrift geschrieben «de raritate luminis» (1740).

Es ist eine äußerst sonderbare Sache, daß es so viele Personen gegeben hat, die die Farben nicht unterscheiden konnten. Ein merkwürdiges Beispiel steht in den Leipziger Sammlungen, I. Band, S. 637. Das sind wahre achromatische Augen. An der Linse und an den Feuchtigkeiten kann es nicht gelegen haben, sondern in der Netzhaut und weiter einwärts. Warum man die Augen und das Gehirn dieser Menschen nicht untersucht? Solche Personen und ähnliche sind Geschenke, die die Natur dem Physiologen macht, und die er nicht annimmt!

Der richtige und vollständige Titel der Leipziger Sammlungen ist: «Sammlungen zur Physik und Naturgeschichte von einigen Liebhabern dieser Wissenschaften. Leipzig, in der Dyckischen Buchhandlung.» Der erste Band erschien 1778, und auf Seite 637 steht: "Joseph Huddart von einigen Personen, welche keine Farben unterscheiden konnten, an D. Priestley.» Es folgt dann ein Auszug aus dem bekannten Originalartikel von Huddart, der in "Philosophical Transactions of the Royal Society of London" (Vol. LXVII, 260, 1777) enthalten ist. Es ist die erste Beschreibung zweier Fälle von Farbenblindheit, die als erster Holmgren wiederaufgefunden hat, denn er erwähnt diese Publikation in seinem 1876 erschienenen Buche «De la cécité des couleurs». Interessant ist, daß Lichtenberg anscheinend die Originalarbeit nicht gelesen hat, sondern durch eine Art Zentralblatt auf sie aufmerksam wurde. Immerhin waren die «Philosophical Transactions» zu seiner Zeit ein ungemein wichtiges Organ, das einem Physiker viel Wichtiges und Wissenswertes vermittelte und Lichtenberg als britischem Gelehrten doch wohl zur Verfügung stand. Wie dem auch sei, die Wichtigkeit und Fundamentalität der Angelegenheit hat Lichtenberg sofort erfaßt, und was er darüber sagt, ist heute so richtig wie damals.

Vielleicht ist gar die Empfindung des Sehens bloß eine Zersetzung des Lichts oder eine Verbindung verschiedener Stoffe unseres Körpers mit diesem einfachen Körper.

Wenn ein Gegenstand, wie z. B. der Mond am Horizonte, durch einen Fehlschluß vergrößert wird, werden auch die einzelnen Teile deutlicher? So daß ich etwa einen Sonnenflecken, den ich bei höherer Sonne nicht würde haben sehen können, alsdann erkennen könnte oder im Monde am Horizonte Dinge sehen, die es unmöglich wäre im Meridian zu unterscheiden.

Wenn man den vollgestirnten Himmel lange ansieht, ohne die Augenlider auch nur einen Augenblick zu schließen, so sieht man endlich gar keine Sterne mehr. Der Versuch ist schwer, weil es etwas schmerzend ist. Sobald man aber nur ein einzigesmal wieder blinzelt und das Auge anfeuchtet, so sind sie alle wieder da. Woher rührt das? 
Von der Trockenheit der äußeren Haut? oder gehen sonst wegen des entstehenden Reizes Veränderungen vor?

Zu Margate in Kent habe ich die See unter der untergehenden Sonne immer erhöht gesehen. War dies vielleicht Irradiation? Was würde das Resultat sein, wenn man mit einer Mikrometerschraube einen weißen Zirkel auf schwarzem Grund und einen schwarzen auf weißem mäße?

Und zum Schlusse aus einem Brief, wahrscheinlich an den Anatomen Sömmering:

Ich habe mein altes Verfahren, die blinde Stelle im Auge auszumachen, sehr verbessert, nämlich ein sehr bequemes Mittel gefunden, den Versuch mit beiden Augen zugleich anzustellen. Ich zeichne zwei schwarze kleine Kreise $a$ und $b$, von anderthalb bis zwei Linien im Durchmesser und in einer Entfernung von 6 bis 7 Zoll voneinander, auf ein Blatt weißes Papier, und in die Mitte zwischen beide mache ich einen kleinen Punkt $c$, allenfalls einen bloßen Stich mit einer Nadel. Hierauf halte ich das Blatt 6 bis 7 Zoll weit vom Auge, gerade vor mich, und beide Augen offen, und sehe mit beiden auf $c$. Damit aber der Punkt $a$ (auf der einen Seite) nicht in das rechte und der Punkt $b$ (auf der rechten Seite) nicht in das linke Auge falle: so halte ich irgend etwas, am bequemsten die konvexe Seite der Schale eines Eßlöffels, hart an die Spitze der Nase, alsdann verschwinden mir beide Punkte, und ich sehe das ganze Blatt weiß, welches sich vortrefflich ausnimmt. Trifft man es nicht gleich, so darf man nur das Blatt entweder dem Auge etwas näherbringen oder mehr davon entfernen; quantum sufficit. Nur muß der Löffel oder was es sonst ist, so gehalten werden, daß man, wenn das linke Auge geschlossen ist, den Punkt $a$, und wenn das rechte Auge geschlossen ist, den Punkt $b$ nicht sieht. Das übrige ist alsdann leicht. Ich habe es sogar mit dem Monde versucht und einmal mit der Sonne, diese leiden wenigstens eine merkliche Veränderung. Sonderbar ist es, daß die Stelle, wo der Punkt verschwindet, doch scheinbar die Farbe des Grundes hat, worauf er steht, und daß man keine Lücke sieht. Aber freilich, was sollte die Lücke für eine Farbe haben? Man sieht gar nichts. Wer schwarz sieht, sieht wirklich etwas. Die Empfindung des Schwarzen ist Gefühl der Inaktivität des Gesichtsorgans. Wenn ich mein $\mathrm{Ohr}$ gegen die Sonne halte, so sieht sie ihm nicht schwarz aus, sondern es sieht gar nichts von ihr. ${ }^{8}$

8 Lichtenberg kannte sicherlich die Modifikation des Mariotteschen Versuches von Picard, die Mariotte selbst beschreibt, und zwar S. 506 seiner Euvres. Picard befestigte nämlich an einer Wand ein Stück Papier und stellte sich etwa $10 \mathrm{Fuß}$ davor auf, während er auf einen dicht vor beide offen gehaltenen Augen placierten Finger so konvergierte, daß die Bilder des Papiers auf beide blinde Flecke fielen und somit das Papier unsichtbar wurde, dieweil es sonst doppelt erschien. Mariotte (S. 516 seiner CEuvres) überbot diesen doch schon schwierigen Versuch und ließ bei binokularer Betrachtung sogar zwei Objekte verschwinden. Er hielt den Daumen so vor sich, daß er dem rechten Auge das linke Papier verdeckte und dem linken Auge das rechte. Fixierte Mariotte nun seinen Daumen, dann verschwanden beide Papiere, weil sie in dem Auge, in welchem sie nicht verdeckt waren, auf die blinden Flecke fielen. Sehr ausführlich geht auf diese Dinge Priestley (S. 191-192 seines Buches "The History and 
Die Zoll und Linien sind wahrscheinlich englische Maße. Ein Zoll hatte 12 Linien und eine Linie mà 2,1166 mm, so daß ein Zoll etwa $25,4 \mathrm{~mm}$ betrug. Die kleinen Kreise hatten daher einen Durchmesser von etwa $4,2 \mathrm{~mm}$ und waren im Mittel $152,4 \mathrm{~mm}$ voneinander entfernt. Um sie auf meine blinden Flecke abzubilden, muß ich sie etwas über 10 Zoll von meinen Augen entfernen. Nun habe ich eine Pupillardistanz von $60 \mathrm{~mm}$, Personen mit größerer Pupillardistanz können eine kürzere Distanz einhalten. Hieraus ist zu schließen, daß Lichtenberg eine große Pupillardistanz gehabt haben muß, was seine Porträts auch beweisen. Ich schätze sie auf Grund von obigen Versuchen und nach einigen Kupferstichen auf über $70 \mathrm{~mm}$. Lichtenberg hatte eine ziemlich schmale Nase mit einer etwas eingezogenen Wurzel, was den Gebrauch eines Löffels unerläßlich macht, bei hohem Nasenrücken genügt es zu seinem Versuch die Hand an die Nase zu halten.

Man könnte einwenden, daß ähnliche Beobachtungen und Aufzeichnungen wohl von vielen gemacht wurden oder doch hätten gemacht werden können, jedenfalls keine Beweise seien für eingehenderes Studium und tieferes Erfassen. Damit würde man aber das ausgehende 18. Jahrhundert und vor allem einen Mann wie Lichtenberg zu Unrecht mit modernem Maße messen. Man notierte damals, und besonders Lichtenberg tat dies, wie sich leicht nachweisen läßt, erst nach reiflichem Überlegen und nie flüchtig, sondern, wie sich zeigt, treffend und scharf formuliert. Man schrieb in dieser Zeit knapp und kurz und setzte außerordentlich viel voraus, da man ja immer zu einem sehr kleinen Leserkreis sprach, den und dessen Kenntnisse man sehr genau kannte. Sehr charakteristisch ist für diese Schreibart auch das Lehrbuch von E rx le b e n, das Lichtenberg später herausgab. Ich will nur zwei Paragraphen hier anführen, nämlich den über die Akkommodation und den über die Presbyopie, die sich im Abschnitt «Vom Lichte» finden.

\section{$\S 387$}

Da das Bild eines entfernten Gegenstandes nicht so weit hinter ein erhobenes Glas und ebenso auch nicht so weit hinter die Kristall-Linse des Auges fällt, als das Bild eines näheren, und wir doch Gegenstände in verschiedenen Entfernungen deutlich wahrnehmen können, so haben wir Grund zu schließen, daß, indem wir nach fernen Gegen-

Present Stade of Discoveries Relating to Vision, Light and Colours", London 1772) ein, welches Buch Lichtenberg natürlich sehr genau studiert hatte und kannte. Er wird aber sicherlich auch Mariottes Euvres, die 1668 erschienen sind, aufs genaueste gelesen haben. 
ständen sehen, entweder die Kristall-Linse unseres Auges näher nach dem Boden desselben zurücke oder auch flacher werde als vorher, oder daß sich endlich der Boden des Auges der Kristall-Linse nähere, und daß bei nahen Gegenständen gerade das Gegenteil geschehe. Ob aber wirklich die Gestalt oder der Ort der Kristall-Linse verändert werde, das ist noch nicht ausgemacht.

\section{$\S 389$}

Wenn das Auge mit zunehmendem Alter nicht allein selbst austrocknet und die Kristall-Linse folglich dem Boden des Auges zu nahe kommt, sondern die KristallLinse auch eben deswegen flacher wird: so können sich nur weit entlegene Dinge auf dem Boden des Auges abbilden, von näheren würde das Bild gleichsam hinter das Auge hinausfallen und auf dem Boden kann also kein ordentliches Bild davon entstehen. Ein solches Auge sieht also auch nur entfernte Gegenstände deutlich, aber nahe nicht und heißt deswegen weitsichtig (presbyta).

Das steht in der zweiten Auflage des «Erxleben» (1777, Seite 294), ist unter Mitwirkung von Lichtenberg entstanden, aber doch noch als das Werk von Erxleben selbst anzusehen. In diesen Zeilen steht nicht nur, was man damals über die Akkommodation dachte, sondern die ganze Geschichte der Akkommodation liegt in ihnen beschlossen. Daß es eine Akkommodation geben müßte, folgt aus der «Theorie des Sehens» von Kepler (1611). Kepler dachte an eine Verschiebung der Linse, desgleichen Pater Scheiner (1619). Eine Formveränderung der Linse nahm Descartes 1637 an, ebenso Peter Camper (1746). Formänderungen des Auges durch den Druck der äußeren Augenmuskeln Sturm (1697), Buffon (1749), Boerhave (1755). Dem Einfluß der Pupillengröße sprach Le Roy 1755 eine große Rolle zu und eine veränderte Hornhautkrümmung bei der Akkommodation glaubte Lobé (Albinus) 1742 nachgewiesen zu haben. Erxleben zitiert nur Häseler (1771), welcher doch wieder an Formveränderungen des Auges glaubte. In diesem Widerstreit der Meinungen ein so klares Bild geben zu können, ist ein Verdienst, das nicht jedem modernen Lehrbuch zugesprochen werden kann, und zeigt, ein wie gründliches Lehrbuch der "Erxleben» in seiner Zeit war.

Mit welcher Gründlichkeit Lichtenberg auch bei Gelegenheitsarbeiten zu Werk ging, zeigt schließlich das letzte Beispiel, welches ich zum Schluß anführen will. Wer es heute zum erstenmal liest, wird weder glauben noch verstehen, daß es der Ursprung aller Arbeiten zur Hygiene des Auges wurde. Der folgende Aufsatz ist, wie fast alle Gelegenheitsartikel von Lichtenberg, im Göttinger Taschenkalender, und zwar 1791, erschienen. Diese Zeitschrift war einem relativ sehr kleinen Leserkreis zugänglich, und sein Inhalt, der durchaus nicht populär war, hatte daher 
von vornherein keine breite Wirkung. Sömmering, der eine allgemeine Verbreitung der Ansichten von Lichtenberg «über die Pflichten gegen die Augen" wünschte, gab daher den folgenden Artikel im Jahre 1793 als Flugschrift heraus. Die Schrift erlebte mehrere Auflagen, wurde ins Französische, Dänische, Schwedische und Holländische übersetzt und fand vor allem in G. J. Beer in Wien einen eifrigen Propagandisten und Nachahmer. Damit war aber die Wirkung dieser Arbeit Lichtenbergs keineswegs erschöpft; in Beers Einkleidung sind ihre Spuren bis gegen 1870 zu finden, und einem Philologen dürften vergleichende Studien, aus welchen so deutlich hervorgeht, wie skrupellos man damals voneinander abschrieb, sicher viel Vergnügen bereiten. Ganz anders Lichtenberg. Er hat Adams wörtlich übersetzt, natürlich wie sogleich folgt, genau zitiert, er hat ihn aber nicht nur verstanden und seine Absichten gefördert, sondern auch Neues hinzugefügt. Dabei lag ihm gar nichts an der Popularisierung seiner Ideen, die Sömmering für ihn besorgte, denn er war kein Arzt und er war zu "aufgeklärt», zu weit über dem Durchschnitt seiner Zeit, dazu war er aber auch zu sehr verwurzelt und beeinflußt von der Zeit seiner Entwicklung, die durchaus im Zeichen der «Enzyklopädie» stand. In der Geschichte der Farbenlehre sagt Goethe über Voltaire: "In der letzten Zeit dieses außerordentlichen Mannes war es zum höchsten Bedürfnis geworden, Göttliches und Menschliches, Himmlisches und Irdisches vor das Publikum überhaupt, besonders vor die gute Gesellschaft zu bringen, um sie zu unterhalten, zu belehren, aufzuregen, zu erschüttern. Gefühl, Taten, Gegenwärtiges, Vergangenes, Nahes und Entferntes, Erscheinungen der sittlichen und der physischen Welt, von allem mußte geschöpft, alles, wenn es auch nicht zu erschöpfen war, oberflächlich gekostet werden.» Dieser Geist war auch in Lichtenberg lebendig, nur daß er gründlich zu Werk ging, jedenfalls so gründlich, als er vermochte, und so ist zu erklären, daß er eines Tages, was er bei Adams gefunden hatte, zusammen mit seinen persönlichen Erfahrungen in einem kleinen Aufsatz der guten Gesellschaft vorlegte. Ich lasse nun diesen Aufsatz, der den Titel hat: «Über einige wichtige Pflichten gegen die Augen», folgen. Die Anmerkungen von Lichtenberg sind durch ein $L$ kenntlich gemacht.

Wie, wenn einmal die Sonne nicht wiederkäme, fragte Amintor'9. Und wie, wenn sie

${ }^{9}$ Der erste Satz ist eine Wiederholung des ersten Satzes von «Amintors Morgenandacht», ein Aufsatz, der vor den «Pflichten gegen die Augen» erschien. Es ist eine 
wiederkäme und ich sähe sie nicht mehr, fühlte noch ihre Wärme, hörte noch den Lobgesang, womit sie der Wald begrüßt, und sähe sie nicht mehr? Ach! dieses ist das Los von Tausenden! Gerechter Gott! Vom Sehenden zum Blinden, welche Veränderung! Der, der noch kaum, gleich einem Gott, den Himmel mit seinem Blick umfaßte, der Sonnen aufzählte zu Tausenden, die Quellen des Lichtes und des Lebens für Geschöpfe ohne Zahl, der in einem $\mathrm{Nu}$ die Frühlingslandschaft mit ihren Blüten und Herden, oder die Pracht der Städte, oder die Wogen des stürmenden Meeres, oder den Ätna und Vesuv, oder Ägyptens Pyramiden übersah, der die Figur der Reiche, ja der Erde selbst maß und zeichnete - da kriecht er nun und ertastet sich mit Mühe in Monaten den kümmerlichen Plan seiner Schlafkammer; die roheste Nachformung von einer Dorfkirche würde ihn Jahre kosten, wenn sie ihm nicht den Hals kostete, und mit einer vom Aetna nur so genau, als das Bild, das im Winkel einer Landkarte Feuer speit, würde er Jahrhunderte zubringen, wenn sie nicht ganz seine Kräfte überstiege; der, der durch das Medium der Gebärden den Menschen im Innersten des Herzens las, hört jetzt bloßes Zungenspiel, der die Wahrheit der Worte wiegen konnte, fühlt jetzt bloß ihre Glätte, und elender, abhängiger Glaube führt die Haushaltung für Selbstüberzeugung in ewiger, ewiger Nacht!

Dieses ist das Los von Tausenden, und wer das Spinngebäude des Organs kennt, auf welches hier alles ankommt, die Menge der Feinde, die ihm von außen und innen drohen, der wird erstaunen müssen, daß es nicht das Los der Hälfte des menschlichen Geschlechtes ist. Bei weitem der größte Teil derer, die dieses Unglück erleiden, die diesen Halbtod, möchte ich sagen, sterben, sterben ihn freilich unverschuldet durch Zufälle, allein keine geringe Anzahl, und zwar gerade unter einer Klasse von Menschen, von denen man es am wenigsten erwarten sollte, ich meine der sogenannten gebildeten höheren Klasse, erleiden ihn öfters durch Schuld, wo nicht wissentlich durch mutwilligen Leichtsinn, doch gewiß sehr oft aus einer Unwissenheit, die leicht zu überwinden gewesen wäre. Für die noch Gesunden dieser Klasse enthält nachstehender Aufsatz Warnung und einigen Unterricht, für die bereits Kränkelnden Unterricht und Trost, wo er möglich ist; für die ganz Erstorbenen findet sich hier nichts; ihre Wiedererweckung, wenn sie möglich ist, gehört für den Arzt ${ }^{10}$. Wie froh würde ich sein, wenn ich durch diese wenigen Blätter nur einem einzigen Leidenden Trost verschaffen, oder nur einen einzigen Leichtsinnigen zur Überlegung bringen könnte, oder jemanden, der nie an den Verlust seiner Augen gedacht hat, dahin, daß er wenigstens daran zu denken anfängt, und sich den Genuß des Lebens nicht vergällt. $O$ man braucht nicht völlig zu erblinden, und kann dennoch von dieser Seite sehr unglücklich sein. Wer je einen Fehler an seinen Augen bemerkt hat, wird wissen, in was für eine Verfassung ihn diese Entdeckung setzte, und was für Zeit die Augenproben wegnahmen. Der Gedanke: in einem Jahre bist du vielleicht blind ${ }^{11}$, mischt sich

kurze philosophische Betrachtung, die mit einem Hinweis auf Brillen und Ferngläser schließt, denn «bei der Brille pflegte ihm öfters einzufallen, daß der Mensch zwar nicht die Macht hätte, die Welt zu modeln, wie er wollte, aber dafür die Macht, Brillen zu schleifen, wodurch er sie schier erscheinen machen könne, wie er wolle." ${ }^{10}$ Sehr charakteristischer Passus. Der Arzt war nur für die ganz Blinden, vor allem für die Kataraktblinden, da.

11 Siehe die Anekdote von Wenzel. 
in alles ein, er ist der erste beim Erwachen und der letzte beim Schlafengehen; keine Gegend und keine Gesellschaft reizt mehr; Nachrichten von neuen Entdeckungen und von neuen Büchern werden mit Unmut gelesen; selbst in Träumen sieht man sich nicht selten im Spiegel durch Augen entstellt, die sich selbst in keinem Spiegel der Welt so sehen könnten. Trifft ein solches Schicksal eine ohnehin hypochondrische Seele, so geht alles viel schlimmer; der vermeintliche Kandidat der Blindheit wird nun wirklich krank, und die reelle Krankheit verschlimmert die halb eingebildete; das Probieren der Augen bei jeder Gelegenheit nimmt zu, und die Proben fallen immer elender aus, so geht es immer crescendo fort bis zur Verzweiflung oder dem Tod. Wer sich also früh einer Augenökonomie befleißigt, erspart sich ein großes Leiden, das, wenn es dennoch kommt, gewiß schon dadurch, zumal bei empfindlichen Seelen, vieles von seiner Bitterkeit verliert, daß es unverschuldet kommt. - Den guten Rat und die Lehren, welche nachfolgende Blätter enthalten, habe ich zum Teil aus einem Aufsatz des Herrn Professors Büsch ${ }^{12}$ gezogen, teils aus einer neueren Schrift des englischen Optikus Adams $^{13}$ und teils aus eigener Erfahrung.

Vor allen Dingen lerne man, auch bei dem besten Gesicht sich nie für ganz sicher zu halten, und ja bei gesunden Augen zuweilen an kranke zu gedenken, und durch behutsamen Gebrauch wenigstens Kraft für sie aufzusparen, wenn sie dereinst alt werden. Man bemühe sich daher soviel als möglich, bei allen Verrichtungen ein gleichförmiges Licht zu erhalten, da wenigstens, wo es leicht angeht und wir von uns abhängen. Eine Vernachlässigung in diesem Artikel ist die schleichende Ursache unzähliger Augenkrankheiten, ja nicht selten der völligen Blindheit. Adams ${ }^{14}$ erzählt bei

12 «Erfahrungen» von J.G.Büsch, Professor in Hamburg. Hamburg 1790, 2 Bände in 80 , im 2. Bande, Seite 261: Guter Rat bei verschiedenen Fehlern der Augen, ein Aufsatz, der sich nicht allein, wie alles, was von diesem vortrefflichen Manne kommt, durch tiefe Einsichten in die Sache überhaupt, sondern über das, welches hier von großem Wert ist, durch Erfahrung und Beobachtungen an sich selbst empfiehlt. $(L)$

J.G.Büsch (1728-1800) war Professor der Mathematik und Direktor der Handelsakademie in Hamburg. Er war myop, was ihn wohl zum Schreiben des zitierten Aufsatzes veranlaßte.

${ }_{13}$ "An Essay on Vision" etc. «intended for the service of those whose Eyes are weak or impaired" by G.Adams. London 1789 , gr. 8. Von diesem Buche ist eine deutsche Übersetzung zu Gotha erschienen. Zweite Auflage 1800. (L)

G. Adams (1750-1795) war "mathematical instrument maker to His Majesty and optician to His Royal Highness the Prince of Wales»; er schrieb: «On the microscope, astronomical and geographical essays, on electricity, on magnetism, geometrical and graphical essays». "On vision" wurde geschrieben «to instruct those, whose eyes are beginning to fail" - und "to do away a general prejudice in favour of spectacles namely, that they act as preservers". Es ist ein Buch von 150 Oktavseiten mit einer sehr guten anatomischen Einleitung, einer geometrisch-optischen Einführung, bei welcher ein Kunstauge zur Demonstration beschrieben wird. Dann folgt eine Brillenlehre, über die später noch einiges gesagt wird, und hierauf einige Bemerkungen über Aphakie, Nachtblindheit und Schielen. Das Buch ist sehr klar geschrieben und war in der Zeit seines Erscheinens sicherlich eine ganz hervorragende Leistung.

14 Seite 101. 
dieser Gelegenheit folgende Geschichte. Ein Rechtsgelehrter in London wohnte so, daß seine Zimmer nach der Straße zu die volle Mittagssonne hatten, seine hintern Zimmer lagen daher nicht allein gegen Mitternacht, sondern gingen auch noch dazu in einen kleinen Hof, der mit einer hohen Mauer umgeben war, und waren also finster. In diesen Zimmern arbeitete er, frühstückte und speiste hingegen in den vordern, in welche ihn überdies sonstige Verrichtungen öfters zu gehen nötigten. Dieses Mannes Gesicht nahm ab, und er hatte dabei einen immerwährenden Schmerz in den Augen. Er versuchte allerlei Gläser, konsultierte Okulisten, aber alles vergeblich, bis er endlich fand, daß der öftere Übergang aus dem Dunkeln zum Hellen die Ursache seiner Krankheit sei. Er veränderte also seine Wohnung, und vermied alles Schreiben bei Licht, und wurde sehr bald wieder hergestellt. Weit trauriger ist der Fall, dessen Herr Prof. Büsch Erwähnung tut: «So manche Augenschwäche», sagt $\mathrm{er}^{15}$, «und völlige Blindheit entsteht bloß aus Verfehlung dieser wichtigen Regel. Als ich vor fünfzehn Jahren den seligen Hagedorn ${ }^{16}$ in Dresden zum ersten Male besuchte, den ich fast ganz blind fand, nahm er meinen Besuch in einem Zimmer an, wo mir das Licht ganz unausstehlich war. Er wohnte in einer ziemlich schmalen Gasse. Das Sonnenlicht fiel von den Quadersteinen der gegenüber gelegenen Häuser scharf zurück in das Zimmer. „Haben Sie', fragte ich, ,in diesem Hause schon lange gelebt?‘ — ,Schon über zwanzig Jahre.“ - ,Und war dies immer Ihr gewöhnliches Arbeitszimmer?‘ — ,Das war es beständig.‘ — ,So', sagte ich ihm, , sehe ich mit Bedauern die Ursache Ihres Unglücks ein, denn in diesem Lichte konnten Ihre Augen nicht gesund bleiben.' - Ich habe», fährt Herr Prof. Büsch fort, «bei mehr als einem Kinde Augenkrankheiten, die vielleicht keinen bösen Ausgang gehabt haben möchten, in einer völligen Erblindung sich endigen sehen, weil deren arme Eltern keine Vorhänge vor den Fenstern und den Wiegen der Kinder hatten.» Vorzubeugen ist hierbei leicht, die Kur des eingetretenen Übels aber oft schwer, ja, wie Adams sagt, und wie es auch wohl bei dem Herrn von Hagedorn der Fall gewesen sein wird, ganz unmöglich. Hieraus wird sich nun leicht auch in dem Zimmer selbst die Lage des Schreibtisches und des Katheders bestimmen lassen. Man schreibe oder lese nie, wenn man es haben kann, in der Lage, daß ein helles Fenster gerade gegenüber so steht, daß jedesmal das Licht in das aufgeschlagene Fenster fällt, sondern lasse das Licht von der Seite einfallen. In Fällen, wo keine solche Abänderung stattfindet, als bei Kanzeln, suche man mit Vorhängen oder sonst auf eine Weise dem Schaden vorzubeugen, und allemal ist es nützlich, es wenigstens zu wissen. Wer weiß, ob nicht, wenn diese Regeln allgemeiner befolgt würden, die schwachen Augen unter die seltenen Krankheiten gezählt werden würden. Als Aufmunterung zur Befolgung dieser Regel muß ich anführen, daß dadurch einige andere, die unten vorkommen werden, Herr Prof. Büsch nunmehr zweiunddreißig Jahre nach

15 Seite 318.

16 Friedrich von Hagedorn (1708-1754), Dichter von Fabeln und Erzählungen, Autor von «Johann, ein munterer Seifensieder». Alles, was nun folgt, ist in die augenärztliche Literatur übergegangen und findet sich bis etwa gegen 1870, oft wörtlich, in zahllosen Lehrbüchern und Schriften. Dabei wird dieser Aufsatz nur ein einzigesmal erwähnt, nämlich in der Zeitschrift von v. Gräfes Vater. Hirschberg hat sie natürlich zitiert. 
dem Zeitpunkte, da er Grund hatte zu fürchten, daß aller Gebrauch seiner Augen aufhören und er im Mittage seines Lebens erblinden würde, noch immer sieht, liest und schreibt. Auch ergibt sich hieraus die Stellung der Betten. Das freie Tageslicht, und noch viel weniger das volle oder reflektierte Sonnenlicht, sollte nie die Augen des Schlafenden treffen können; denn selbst wenn es, ihm unbewußt, während des Schlafes auf die Augenlider fällt, so kann dieses, zumal, wenn er bereits schwache Augen hat, den ganzen Tag über die größten Beschwerden verursachen. Hierauf hat man besonders auf Reisen zu sehen, und wenn man des Abends spät ankommt, die Lage der Fenster und die Beschaffenheit der Bettvorhänge zu untersuchen, damit man nicht auf eine unangenehme Weise des Morgens vom Tage oder gar von der Sonne überfallen werde. Im Wagen, wo die hellen Fenster sehr stark gegen das Übrige abstechen, ist ein doppelter oder dreifach zusammengenähter, grüner Flor für empfindliche Augen das beste Hilfsmittel, denn die Läden hemmen den Umlauf der reinen Luft, und die feinsten Vorhänge die Aussicht, die, zumal auf entfernte Gegenstände, dem Auge in vieler Rücksicht so wohltätig ist. Einfache Flöre, dergleichen die Damen tragen, um dadurch zu sehen, und gesehen zu werden, sind zu dünne, und wenn sie geblümt sind, noch eher schädlich. Aus dieser ersten Regel, überall nach gleichförmigem Lichte zu trachten, ergibt sich auch die Beschaffenheit der Schirme. Man gibt dem schwachen Auge gern einen Schutz von oben, dieses ist sehr recht getan, sagt Herr Prof. Büsch, in so fern dadurch das helle, von oben einfallende Tageslicht von dem Auge abgehalten wird. Aber man bedenkt nicht, daß dadurch die untere Hälfte des Auges, in welche das Licht von oben fällt, ganz in Schatten gesetzt, die obere Hälfte aber beständig durch das in dasselbe fallende Licht gereizt wird. Dies ist keinem Auge gut. Es muß ein sehr gesundes Auge sein, das dabei lange aushält. Wie aber, wenn das Ubel gar mehr im obern Teile des Auges seinen Sitz hat? Dann ist es gerade verkehrt gehandelt. Der gesundere Teil wird geschützt und der schwächere soll immerfort Dienste tun ${ }^{17}$. Überhaupt erfordert alle Erleichterung, die man dem Auge durch Dunkelheit verschafft, viele Vorsicht. Alle am Tage selbst mit grünen Vorhängen erkünstelte Verdunkelung kann schädlich werden, teils weil sie nie so vollständig erhalten werden kann, daß nicht hie und da etwas durchschimmere, teils weil man, wenn man nicht ganz müßig oder unfähig ist, sich zu bewegen, unmöglich lange darin aushalten wird. Die natürliche Dämmerung ist die beste, und man sollte den Genuß derselben dem ermüdeten Auge nicht mißgönnen, zumal da sie außerdem der Überlegung so sehr günstig ist. Schreiben oder lesen muß man in der Dämmerung nicht. Es ist ein Verfahren, das, den gelindesten Ausdruck zu gebrauchen, töricht ist. Der schnöde Gewinn an öl und Zeit geht tausendfach durch das Leiden und den Unmut hin, den man sich durch schwache Augen zuzieht. Ein Freund von mir klagte mir eines Tages: er habe sonst so schön in der Dämmerung lesen können, jetzt könne er es nicht mehr, und fürchte, wenn es mit dieser Abnahme seines Gesichtes so fortginge, so würde er vor seinem vierzigsten Jahre blind werden. Ich sagte $\mathrm{ihm}$, er habe freilich recht, ich glaube auch, daß, wenn es so fortginge aber mit dem Lesen in der Dämmerung, so würde er blind werden. Er habe sehr richtig geschlossen, ob er gleich die Wirkung für die Ursache genommen habe, er könne nicht deswegen, sagte ich, nicht mehr in der

${ }^{17}$ Seite 323 . 
Dämmerung lesen, weil sein Gesicht im Abnehmen sei, sondern es nehme ab, weil er immer noch in der Dämmerung lesen wolle. Sein Fehlschluß, so sehr er auch sonst Fehlschlüsse haßte, machte ihm dieses Mal keine geringe Freude. Er unterließ das Lesen in der Dämmerung, und sein Gesicht nahm so wenig ab, daß ich diese Geschichte auch mit deswegen hieher setze, um ihm, der diese Zeilen in diesem kleinen Druck, jetzt in seinem fünfzigsten Jahre gewiß (vielleicht gar einmal aus Mutwillen in der Dämmerung) lesen wird, eine Freude in der Ferne zu machen. Es ist überhaupt ein sehr großer, wiewohl sehr gemeiner Irrtum zu glauben, ein schwaches Licht sei den Augen günstig. Dem unbeschäftigten Auge wohl, das nicht sehen will, allein dem sehenwollenden ist es schlechtweg schädlich und ein starkes zuträglicher. Daß hier die Rede nicht vom unmittelbaren Sonnenlichte oder von weißen Gegenständen, als z. B. von Schnee zurückgeworfenem, ist, versteht sich von selbst. Dieses kann freilich Entzündungen der Augen bewirken, die nicht bloß Schwäche des Gesichtes, sondern völlige unheilbare Blindheit in kurzer Zeit zur Folge haben können. Gegen einen solchen Mißbrauch des Lichtes warnt aber auch die Natur gemeiniglich bald durch ihr gewöhnliches Mittel, den Schmerz, und das unerträgliche, was jene Empfindung begleitet. Was man gemeiniglich schädlich im starken Lichte zu finden glaubt, ist nicht sowohl dieses, als der Mangel an gleichförmiger Verbreitung desselben im Auge. Man kann am Tage ohne die mindeste Beschwerde stundenlang in den Mond sehen, selbst wenn er hoch über dem Horizont steht, bei der Nacht geht dieses nicht an, ja man hat Beispiele, daß Astronomen, die ihn des Nachts durch Ferngläser lange unverrückt und ohne gefärbte Gläser betrachtet haben, um ihr Auge gekommen sind. Dieses rührt daher: Am Tage leuchtet nicht bloß der Mond, sondern auch alle Gegenstände umher, und selbst der benachbarte Himmel wirft blaues Licht zurück. Dadurch wird die Pupille gehörig verengert, überflüssiges Licht abgehalten, und überdies der Boden des Auges mit gleichförmigem übermalt. Hingegen bei der Nacht wirken die Gegenstände sehr ungleichförmig auf das Auge, und bringen daher in einander naheliegenden Teilen desselben entgegengesetzte Wirkungen, teils gleichzeitige, teils sukzessive hervor, welches immer eine Art von anfangs zwar vorübergehender, aber endlich mehr oder weniger anhaltender Zerrüttung ist, derjenigen analog, die plötzlicher Übergang von Hitze zur Kälte dem Leibe verursacht. Man findet daher schon wirklich in obigem Falle einige Erleichterung, wenn man das Objektivglas erleuchtet, da noch mehr Licht auf das Auge fällt als vorher, da der Mond allein da war, allein es ist nun alles gleichförmiger, der Mond scheint nicht mehr an einem schwarzen, sondern an einem weißlichen Himmel zu stehen. So würde das Blatt, worauf ich schreibe, das mir mit so sanftem Licht zu leuchten scheint, unerträglich zu glühen scheinen, wenn es dieses erborgte Licht des Nachts in einem übrigens dunkeln Zimmer, als sein eigenes zurückwürfe. Ich würde glauben, auf weißglühendes Blech zu schreiben und mit der Federspitze einzelne Stellen abzulöschen. - Also, wenn es dann doch einmal bei Licht gelesen oder geschrieben sein soll, so ist es immer besser, zwei oder drei Lichter zu gebrauchen, als ein einziges, nur muß die Flamme selbst mit so wenigem Aufwand von Schatten verdeckt werden, als es die Ümstände verstatten.

${ }^{18}$ Segners Lampe war eine öllampe mit flachem Volldocht und einem Schirm. Sie ist u. a. in Ph. Heinekens "Ophthalmobiotik" (1815) als Studierlampe beschrieben. J.A.von Segner (1704-1777) war Professor der Mathematik und Medizin in Göttingen. 
Herr Prof. Büsch hält zu dieser Absicht die kleinen Taschenschirme aus Taffet für die bequemsten und besten, deren Mangel man auch ebenfalls mit einer Karte ersetzt, die man vermittels einer Haarnadel befestigt. Die Lampen mit Schirmen, die, wie die Segner18schen und andere ähnliche, das ganze Zimmer verfinstern, bis auf die Stelle, da man liest, müssen bei fortgesetztem Gebrauch notwendig das beste Gesicht durch eben diese ungleiche Verteilung des Lichtes schwächen, da bei jedem Umhersehen das Auge die Veränderung erleidet, von der wir oben geredet haben, und auch selbst in dem Falle, da man nicht umhersieht, jene ungleiche Erleuchtung des Inneren des Auges bewirkt, die so schädlich ist. Schade, daß die vortreffliche Lampe des Argand ${ }^{19}$, die sonst in aller andern Rücksicht eine der schönsten Erfindungen ist, auch diesen Fehler hat. Der Erfinder hat zwar einigermaßen dieser übeln Wirkung dadurch vorzubeugen gesucht, daß er die Schirmstürze aus dickem, weißem Papier macht, wodurch das Licht mehr durch die Stube verteilt wird, und freilich nicht so schädlich wie ein undurchsichtiger Schirm oder wie der Anblick der Flamme selbst wird, aber doch noch immer zu abstechend gegen das übrige Licht des Zimmers, weil die Lichtflamme bei dieser Lampe so äußerst lebhaft ist. Auch hat man den Rauchfang aus gefärbtem Glas gemacht, dadurch wird aber ein Teil der Absicht dieser Lampe verfehlt, nämlich die große Helle. Daß Schirme, die man über den Kopf stürzt, das Licht im Auge ebenfalls ungleichförmig verteilen, ist schon oben erinnert worden.

Der zweite Hauptrat ist: Man muß den Augen nie mehr anmuten, als sie vertragen können, und die Art und die Zeit der Beschäftigungen soviel als möglich nach dem Zustande der Augen wählen²0. Man muß also, soviel als möglich, alle lange anhaltende Anstrengung der Augen vermeiden, und in den Beschäftigungen abwechseln. Zum Glück werden die von Nerven herrührenden Augenschwächen gewöhnlich solchen Menschen zuteil, die dieses noch können, und seltener Leuten, die in körperlichen oder in leichteren Handarbeiten sich anhaltend beschäftigen. Herr Prof. Büsch enthält sich seit vielen Jahren alles anhaltenden Lesens bei Licht, und wählte dafür lieber das Schreiben, weil er dann seinen Augen noch durch den Gebrauch des blauen Papiers zu Hilfe kommen kann. Weil mir aber, setzt er hinzu, meine gesetzten Arbeiten nicht Beschäftigung genug gegeben hätten, so mußte ich mich nach anderen Gegenständen umsehen. Kurz, dieser Umstand insonderheit habe ihn erst spät zum Schriftsteller und nun beinahe zum Vielschreiber gemacht. Mancher Ausländer wegen, - denn der Almanach21 wird übersetzt, muß ich hinzusetzen - und zwar zu einem, der der Nation Ehre macht. Soviel Trost diese Geschichte dem Denker gewähren wird, der aus sich selbst schöpfen kann, so wenig Tröstliches enthält sie für den Kompilator, der seine

10 Argandsche Lampe, beschrieben 15. Januar 1785 im Journal de Paris als elampe à cheminée». Es ist eine Rundbrennerlampe. Argand hatte als erster die Idee, die Leuchtfläche der ölflamme dadurch zu vergrößern und zu verbessern, daß der flache Docht der Länge nach kreisförmig in der Doppelwand eines metallenen Hohlzylinders zusammengebogen wird, so daß trotz der runden Form im gewissen Gegensatz zum alten runden Volldocht neben der äußeren auch eine innere Luftzufuhr möglich wurde. Aimé Argand (1755-1803).

20 Büsch, Seite 333.

21 Der Göttinger Taschenkalender erschien deutsch und französisch. 
Bibliothek oder gar die öffentliche mit zu seinem Kopfe rechnet, und bei welchem sich besinnen, nachschlagen heißt. Doch diese gehören mit unter die subtilen Handarbeiter, von denen wir soeben gesagt haben, daß sie nicht so leicht mit dieser Krankheit befallen werden. Wer sich vorlesen lassen und diktieren kann, kann sich freilich große Erleichterung verschaffen, und allen anstrengenden Gebrauch der Augen bloß auf den Tag versparen, mit sehr großem Gewinn für dieselben.

Dritter Rat: Man beschäftige seine Augen in freien Stunden soviel als möglich in freier Luft und im Sehen in die Ferne, man wähle seine Vergnügungen in dieser Rücksicht. Reiten hat einen längst erkannten Nutzen für nervenschwache Augen, durch die heilsame Erschütterung der Nerven. Fahren und Spazierengehen haben ihn auch in dieser Rücksicht. Von allen aber ist dieses der Hauptvorteil, den sie dem schwachen Auge verschaffen, daß dasselbe mit einer Menge von Gegenständen beschäftigt wird, deren keiner das Auge lange auf sich zieht, und die in der Entfernung, worin man sie sieht, demselben ein hinlänglich sanftes Licht zusenden.

Zum Trost bei anhaltender Augenschwäche dient die Bemerkung, daß sie sich selten mit völliger Blindheit endigt, zumal wenn man sich der erwähnten Vorsicht bedient, und man lasse sich daher nicht gleich durch Okulisten ${ }^{22}$ schrecken. Es gibt unter ihnen sehr seltsame Menschen, die alle die prachtvolle Windigkeit des Ritters Taylor ${ }^{23}$ ohne seine Geschicklichkeit besitzen. Ich kann hier aus eigener Erfahrung reden, und ergreife mit Vergnügen diese Gelegenheit, einem Manne ein kleines Denkmal zu stiften, das ich ihm schon längst zugedacht habe, ohne die Gelegenheit dazu finden zu können. Dieser Mann ist der berühmte Okulist Wenzel ${ }^{24}$, der Vater, in London. Wer ihn noch nicht kennt, kann die kurze aber brillante Geschichte seines eigenen Wertes, mit stehenbleibenden Schriften gedruckt, in jedem englischen Morning paper lesen. Wenn schon die Gleichzeitigkeit einem Geschichtsschreiber so vielen Kredit gibt, so kann man leicht denken, was gar diese Geschichte sein müsse, da er selbst der Verfasser davon ist. $\mathrm{Zu}$ diesem wackeren Landsmanne verfügte ich mich im Jahre 1775, da sich ein Zufall an einem meiner Augen25 zeigte, der einigen meiner Freunde and besonders mir sehr bedenklich schien. Er wohnte in einer der ersten Straßen

${ }^{22}$ Als Okulisten bezeichnete man damals die reisenden Starstecher. Hirschberg nennt sie die «irrenden Ritter der Augenheilkunde». Sie spielten im 18. Jahrhundert eine außerordentliche Rolle. Nicht mit Unrecht bezeichneten Zeitgenossen ihr 18. Jahrhundert als das Säkulum der Okulisten. Ein Hauptrepräsentant war Taylor.

Ebendaselbst Seite 336.

23 Chevalier John Taylor, 1703 in Norwich geboren, studierte in Leiden, bereiste ganz Europa und starb 1772 in England. Er reiste in einer mit Augen bemalten Kutsche, auf der stand: qui dat videre, dat vivere. Sein Buch "The History and Adventure of the Chevalier John Taylor, Ophalmiatros, written by him self and adressed to his son" (London 1761) ist ein amüsantes Buch, wirklich voll prachtvoller Windigkeit.

24 Michael Baron von Wenzel war ein Deutscher, später seßhaft in Paris, lebte und wirkte auch oft in London, wo er 1790 starb. D. Chodowiecki hat seine Staroperation in Kupfer gestochen. Wenzel operierte mit seinem noch heute zur Exenteratio bulbi von manchem verwendeten dreieckigen Messer nach unten, hat aber wohl auch erstmalig den Lappenschnitt nach oben ausgeführt. Er war trotz aller Fehler und Schliche doch ein Mann von Verdienst. 
Londons, in Pall-Mall, da, wo nachher auch Graham ${ }^{26}$ seine himmlische Bettlade aufschlug. Bei dem Eintritte in das Haus wurde ich von einem Paar Bedienten oder Lehrlingen, denn sie hatten in ihrem Betragen etwas von beiden, mit den Augen gemessen und gewogen, vermutlich zu erforschen, ob ich ein solventer oder Gratis-Patient sei, denn in meinem Anzuge mochten sie wohl auch so etwas von beidem entdeckt haben. So kam ich endlich vor Herrn Wenzel, der mit jemandem in der Stube ein sehr breites Englisch sprach. Ich fragte ihn auf die bescheidenste Weise von der Welt auf Englisch, ob ich wohl deutsch mit ihm reden könne, denn es gibt in England Deutsche, die es nicht gern Wort haben wollen, daß sie es sind. 0 , sagte er, sprechen Sie mit mir, was für eine Sprache Sie wollen. - Dieses gab mir eine sehr hohe Idee von den Sprachkenntnissen dieses Mannes; ich klagte ihm also mein Anliegen deutsch. Er ließ mich niedersitzen, besah mein Auge mit sehr bedeutendem, liebreichem Kopfschütteln, und auf die Frage, was er von dem Umstand hielte, sagte er: Sie werden blind. - Können Sie mir aber wohl helfen? - 0 ja. - Und was muß ich Ihnen dafür bezahlen? - Zehn Guineen, war die Antwort; ich gebe Ihnen etwas in einem weiten Glase, da halten Sie das Auge des Tages etliche Male hinein, usw. Ein feiner Charlatan war denn doch der Mann nicht. Er hätte mich bloß niederschlagen sollen, allein der unanständige dezisive Ton seiner Worte richtete mich mehr auf, als mich ihre Bedeutung niederschlug, und ich sah auf einmal, wen ich vor mir hatte, bezahlte ihm eine halbe Guinee für die gemachte Freude, und ging nach der Straßentür zu, wohin er mich mit bezahlter Höflichkeit unter vielen Bücklingen begleitete. Vollkommen tröstlich für mich war indessen diese Unterredung im ganzen nicht, denn: ich hörte nachher von Wenzels Talenten wenigstens nicht immer schlecht sprechen. Indessen nahm nun bald meine Geschichte eine andere Wendung. Auch hier muß ich ein paar Männer nennen, nicht um ihnen ein Denkmal zu stiften, denn dieses haben sie, die sehr weit über alles Lob, das ich ihnen erteilen könnte, erhaben sind, selbst längst getan. Ich sprach nämlich von meinem Zufall an einem hohen Orte. Die Folge war, daß der königliche Wundarzt Hawkins ${ }^{27}$ zu mir kam. Bei seinem Eintritt in die Stube war es, als gingen Zutrauen und Hilfe vor ihm her, mit so liebreichem Ernste nahte er sich mir. Er sah mir lange in das Auge, aber ohne Kopfschütteln, gab mir alsdann die Hand, und sagte mit unbeschreiblich sanftem Ton, den ich noch immer höre: Seien Sie ganz ohne Sorgen, Sie haben nichts zu befürchten, und verordnete mir ein

25 Aus den Briefen Lichtenbergs geht hervor, daß er häufig «augenkrank» war. Seine Angaben sind aber in den Briefen noch ungenauer als hier, so daß nichts Näheres über diese Krankheit ausgesagt werden kann. Richter, der der Gewohnheit seiner Zeit entsprechend bei seinen Krankengeschichten den Namen des Kranken immer voll ausschreibt, erwähnt, soweit ich finden konnte, Lichtenberg nicht.

26 G. Graham (1675-1751), ein wegen seiner Geschicklichkeit sehr bekannter Uhrmacher.

27 Caesar Hawkins (1711-1786) ; er war Sergant-surgeon Georgs II. und Georgs III. von England. Drei seiner Nachkommen folgten ihm in diesem hohen Amte nach. Lichtenberg hatte als königlich-großbritannischer Hofrat Zutritt zum königlichen Hof, wurde dem König Georg III. vorgestellt und einige Male von ihm in London empfangen. So auch, als er Wenzel konsultierte. 
sehr leichtes Mittel, das mir ein paar Groschen kostete. Als ich bald darauf nach Göttingen kam, fing ich doch wieder an zu sorgen, denn die Augenkranken sind gar vorsichtige Menschen, und fragte unseren jetzigen Herrn Leibarzt Richter ${ }^{28}$. Hier erhielt ich dieselbe herzliche Versicherung mit denselben Mitteln, und seit der Zeit hat das Übel, das doch schon zu dem Grade angewachsen war, daß es die Hornhaut durch Andruck etwas verstellte, und ich wirklich mit diesem Auge doppelt sah, nicht allein nicht zugenommen, sondern ist so völlig verschwunden, daß ich noch kaum im Vergrößerungsspiegel die Spur davon finde. Dieses zeigt, wie man die Augenärzte wählen müsse, deutlich. Die Regel gilt auch bei der Wahl der Ärzte überhaupt. - Ehe ich nun za den Hilfsmitteln schreite, die das Gesicht von Gläsern hoffen kann, und der dabei nötigen Vorsicht, so schreibe ich Herrn Adams, einem erfahrenen, vorsichtigen Manne ein äußeres Mittel nach, das allemal ohne Schaden und oft mit Vorteil gebraucht worden ist, wo sich eine Schwäche der Augen früher, als man vom Alter des Patienten erwarten sollte, einstellt, und wovon auch sonst keine in die Augen fallende Ursache vorhanden ist ${ }^{29}$. $\mathrm{Zu}$ einem halben Quartier Branntwein tut man zwei Unzen Rosmarinblätter in eine schwarze Flasche und schüttelt alles drei Tage hintereinander etliche Male des Tages durcheinander, läßt es drei Tage stehen, und seiht es alsdann durch. Von dem Klaren dieses Ausgusses mischt man sodann einen Teelöffel voll mit vier Teelöffel voll warmen Wassers und wäscht damit beim Schlafengehen die Augen so, daß man die Augenlider jedesmal in eine solche Bewegung setzt, daß dabei etwas von dem Ausguß zwischen das Augenlid und den Augapfel kommt. Nach und nach kann man immer weniger Wasser nehmen, bis man endlich mit gleichen Teilen von jedem beschließt -

Allein aller Mühe und Vorsicht ungeachtet, wird oft das Auge schwächer, so wie die Stärke der körperlichen Hülle zu sinken anfängt, oder leidet wenigstens Veränderungen, die eine Beihilfe nötig machen. Doch ist dieses nicht immer eine notwendige Folge des Alters, ob es gleich eine sehr gewöhnliche ist. Herr Prof. Büsch redet von einer Frau, die, als er seinen Aufsatz schrieb, noch in Hamburg lebte, die in ihrem hundertundzehnten Jahre noch eines vollkommenen Gesichtes genießt; und ähnliche Beispiele gibt es im siebzigsten und achtzigsten Jahre gewiß unzählige, und würden gewiß noch häufiger sein, wenn man von den Jahren des reifenden Verstandes an eine gehörige Gesichtsökonomie bei sich eingeführt hätte. Ist es aber nun einmal nicht anders, stellen sich die Folgen des Alters beim Gesicht ein, so affektiere man nicht lange eine Kraft, die einem nicht mehr natürlich ist. Durch Affek-

28 A. G. Richter (1742-1812) war seit 1766 Extraordinarius in Göttingen und hat dort die erste Augenklinik eingerichtet. Er gehörte zu den besten Augenärzten seiner Zeit.

${ }^{29}$ Siehe Seite 102 (Adams). Nach den Angaben von Adams: etwa 55 g Rosenblätter zu etwa einem halben Liter «brandy". Nicht uninteressant ist, daß der vorsichtige Adams, ehe er sein Rezept gibt, sagt: "I hope I shall not be thought to have stepped improperly out of the line of my professio, in recommending the following remedy", und zwar soll man sie nur anwenden, wenn sich eine Schwäche usw. einstellt, "and withoud any disease or other apparent cause, if it does not answer the purpose, no ill will attend the use of it". Kurz gesagt: es hilft auch nicht. Ich konnte nicht eruieren, woher Adams sein Rezept hatte. 
tation von Kraft in gewissen Jahren geht nicht selten auch noch der Teil derselben verloren, den man noch hat, ohne daß man sonst etwas dabei gewänne. Daher sind auch die geraden offenherzigen Leute, die nicht um ein Haar stärker oder gesünder sein wollen, als sie sind, diejenigen, die am längsten aushalten. So empfängt auch hier die Tugend ihren Lohn durch sich selbst.

Man kann überzeugt sein, daß dieser Fehler der Augen $^{30}$ eintreten werde, oder bereits eingetreten sei:

1. Wenn man genötigt ist, um kleine Gegenstände deutlich zu sehen, sie in einer beträchtlichen Entfernung vom Auge zu halten.

2. Wenn man (des Abends) mehr Licht nötig hat als sonst und z. B. um deutlich zu sehen, die Kerze zwischen den Gegenstand und das Auge bringen muß. (Ein in aller Rücksicht äußerst schädliches Verfahren, wenn damit angehalten wird.)

3. Wenn ein naher Gegenstand, den man mit Aufmerksamkeit betrachten will, sich zu verwirren und wie mit einem Nebel zu überziehen anfängt.

4. Wenn die Buchstaben beim Lesen zuweilen ineinanderzufließen und doppelt und dreifach zu sein scheinen.

30 Wörtliche Übersetzung aus Adams mit Ausnahme der eingeklammerten Worte bis zu dem Satze «Brillen sind Krücken und Konservier-Krücken, usw. Bei Adams steht Seite 105: «ant it» (nämlich Brillen zu tragen ohne Notwendigkeit) «would be as absurd, to advise the use of spectacles to those who feel none of the foregoing inconveniences, as it would be for a man in health to use crutches to save his legs». Der vieldeutige Satz, daß Brillen Krücken sind, stammt von Lichtenberg, der als Physiker natürlich nicht wissen konnte, daß ihm dieser Satz bis zum heutigen Tage nachgebetet wird und zwar gerade in der falschen Interpretation, die er bestritt. Denn Adams und Lichtenberg richten sich gegen die Konserviergläser und die «visual spectacles». Erstere gehen zurück auf H. Sirturus, der 1618 Schutzgläser ohne Brechkraft erfand (siehe Rohr, «Die Brille als optisches Instrument», 1921). Später kam dann die Röhrenbrille auf, die wohl den Übergang zu den «visual spectacles» bildete. Diese hat B. Martin 1756 erfunden, es waren nach Rohr gewöhnliche gleichseitige Gläser, deren ursprüngliche öffnung von $3,8 \mathrm{~cm}$ durch einen breiten, das Blickfeld beschränkenden Hornrand auf $2,5 \mathrm{~cm}$ vermindert wurde. Diese Gläser waren anscheinend sehr Mode und galten als Zeichen von Stand und Würde, was ja noch heute im fernen Osten der Fall zu sein scheint. Es ist dies wahrscheinlich eine sehr alte spanische Erbschaft. Denn in einem 1699 in Amsterdam erschienenen Buche, *Het leven en bedrijf van den Heere Michael de Ruiter Hertog, Ridder en LuitenantAdmiraal van Holland en West-Vrieslandt", geschrieben von Gerard Brandt, wird S. 286 erzählt, daß der Unterköning von Valencia den Admiral de Ruiter festlich empfing und zum Willkomm bei Kanonengedröhn und Trompetenschall «een bril op de neuze, naar't gebruik der Spanjaarden, tot betoog van deftigheit" (eine Brille auf der Nase, nach der Sitte der Spanier als Zeichen seiner Vornehmheit) trug.

Die Mode nahm im ausgehenden 18. und beginnenden 19. Jahrhundert anscheinend so überhand, daß die Augenärzte den «moedwillige brillendragers», wie sie Mensert 1831 in seinen «Verhandelingen over de brillen» nennt, recht ernst ins Gewissen zu reden nötig achten. 
5. Wenn die Augen nach einer mäßigen Anstrengung gleich so sehr ermüden, daß man genötigt ist, zur Erholung auf andere Gegenstände zu sehen.

Bemerkt man einen oder mehrere von diesen Umständen, so ist es Zeit, sich nach Gläsern umzusehen, die alsdann, gut gewählt, den Augen zur mehreren Erhaltung, ja zur Heilung dienen können, die sonst durch unnütze Anstrengung, deutlich zu sehen, noch mehr verdorben werden würden. In diesem Verstande können die Brillen wirklich Konserviergläser werden. Man muß aber ja nicht glauben, wie sehr gewöhnlich geschieht, daß es Gläser gebe, die ein noch völlig gesundes Gesicht zu konservieren dienen. Brillen sind Krücken, und Konservier-Krücken für gesunde Beine gibt es nicht und braucht man nicht. Je eher man dazu tut, desto besser. Jeder Aufschub verschlimmert die Sache. Adams führt einen Fall an, da eine Dame aus falscher Scham den Gebrauch der Brillen so lange aufschob, daß man ihr am Ende nur noch mit Gläsern von solcher Dicke und Brennweite, dergleichen man am Star operierten Personen zu geben pflegt, eine leidliche Hilfe verschaffen konnte; da hingegen Personen, die beizeiten Gläser von großen Brennweiten gebrauchten, öfters imstande gewesen sind, ihre Brillen beiseite zu legen und mit den bloßen Augen zu sehen. Man sei daher bei der Wahl, zumal der ersten Brille, sehr auf seiner Hut, und wende sich an erfahrene Leute. Man wähle keine starken Vergrößerer, sondern nur solche, durch welche man mit Leichtigkeit in eben der Entfernung lesen kann, in welcher man sonst mit Bequemlichkeit ohne Brillen zu lesen pflegte. Wird freilich das Auge noch flacher ${ }^{31}$, so muß man stärkere Vergrößerer suchen, aber sich immer hüten, nicht plötzlich zu weit zu gehen. Eine gute Probe, daß man zu weit gegangen und seine Brillen zu stark gewählt habe, ist, wenn man das Buch näher ans Auge bringen muß, als sonst Personen von gesunden Augen zu tun pflegen (nämlich näher

31 Das Flachwerden des Auges ist wohl am besten zu verstehen aus den folgenden Zitaten aus Adams (Seite 89 und 91 im Abschnitt "Of long or old-sighted eyes»): «Various are the causes which may occasion this defect, if the convexity of the cornea be lessened, or if either side of the crystalline becomes flatter, this effect will be produced: if the retina be removed too far from the cornea or crystalline it will give rise to the same defect as will also a less refractive power in the pellucid parts of the eye, in like manner, too great aproximity of the objets or too small a pupil, will prevent the rays from uniting till they are beyond the retina, but if all these causes concur together, the effect is greater. This defect is, howewer, in general attributed to a shrinking of the humours of the eyes, which causes the cornea and crystalline to lose their original convexity, and to become flatter.

Increasing years have a natural tendency to bring on this defect and earlier among those, who have made the least use of their eyes in their youth, but whatever care be taken of the sight, the decays of nature can not be prevented: the humours of the eye will gradually waste and decay, the refractive coats will become flatter and the other parts of the eye more rigid and less pliable."

Ich glaube, daß das Flacherwerden noch weiter zurück zu verfolgen ist, denn bei Newton, "Optics» (1718), Seite 13 steht: "If the humours of the eye by old age decay, so as by shrinking to make the cornea and coat of the crystalline humour grow flatter than before, the light will not be refracted enough», usw. 
als neun bis acht Zoll). Zuweilen ereignet es sich, daß Personen, die am Tage gut und bequem durch die Brille lesen können, bei Licht aber nicht, wenigstens nicht ohne beschwerliche Anstrengung; diese werden wohl tun, wenn sie sich eine etwas mehr vergrößerende anschaffen, die sie nur bei Licht gebrauchen. Man hüte sich vor den sogenannten Brillen mit Bedeckungen oder Blendungen, die die Engländer evisual spectacles» nennen, deren Gläser, von geringer Apertur, mit sehr breiten schwarzen Ringen, gewöhnlich aus Horn eingefaßt sind. Ein unwissender Mann ${ }^{32}$ hat ihnen aus einem mißverstandenen principio diese Einrichtung gegeben, die bei Fernröhren nötig, hier aber nicht bloß unnütz, sondern schädlich ist, eben wegen dieser starken und nahen Schatten, und weil bei etwas langen Zeilen der ganze Kopf in Bewegung gesetzt werden muß. Ebenso unnütz und schädlich, wiewohl nicht in ganz so hohem Grade, sind die grünen Brillen. Herr Prof. Büsch sowohl als Adams sprechen aus Erfahrung stark dagegen. Das Grüne ist allerdings eine sanfte und angenehme Farbe, aber nicht die Farbe der Gegenstände, die man durch grüne Brillen ansieht. Sie geben allen Farben, das Weiße und Grüne ausgenommen, ein unangenehmes und schmieriges Ansehen, und werden sie abgenommen, welches der Fernsichtige bei fernen Gegenständen tut, so erhalten die Gegenstände ein blendendes, anfangs sogar rötliches Ansehen, welches den Augen schadet ${ }^{33}$. Auch in dieser Erfindung also ist mehr guter Wille als Verstand. Die Furcht und Scham, alt zu scheinen, denen wir den ganzen zweiten Teil der kosmetischen Kunst zu danken haben, haben ebenfalls an den Krücken gekünstelt, wodurch sich das alternde Gesicht forthelfen muß oder ihnen wenigstens das Ansehen von einem Spazierstock zu geben gesucht, den man mehr aus Laune als Not gewählt hätte. Sie haben nämlich das Auge zu bewaffnen gesucht, ohne die Nase zur Waffenträgerin zu machen, und die sogenannten Lesegläser erfunden, die man in der Hand hält. Die Absicht dieser Gläser soll sein, sich bei der Fernsichtigkeit des Alters noch ein Ansehen von Jugend zu geben; dann soll die Würde des Gesichtes nicht so sehr darunter leiden, und endlich auch die Nase nicht gemißbraucht werden und den guten Ton nicht verlieren. Der erste Vorteil ist gewiß sehr gesucht und würde wegfallen, sobald man dergleichen Gläser nur bei Alten sähe. Was den zweiten Vorteil betrifft, so ist zwar nicht zu leugnen, daß zu allen Zeiten und bei allen Völkern die meisten Handlungen, worin sich die Nase entweder von selbst mischt, oder in welche sie mit Gewalt gezogen wird, sobald sie nicht mit zu den Geruchsgeschäften gehören, ein etwas lächerliches Ansehen gewinnen. Dahin gehört z. B. das Tragen großer Warzen darauf, die gar für die Nase nicht gehören; das Umschlagen von Blättern in Büchern, das Auffangen und Parieren von Schlägen, denen sie nicht gewachsen ist, oder wenn sie sich gar zum Zügel oder zur Handhabe gebrauchen läßt, ihren Besitzer daran herumzuführen. Allein nichts, was die Nase zur Unterstützung der Augen tut, hat sie je lächerlich gemacht, wegen der bekannten Verwandtschaft, die zwischen beiden stattfindet. Es ist nämlich bekannt, daß beide schon in der frühesten Jugend gemeiniglich zugleich weinen, ja daß selbst im Alter die Augen noch übergehen, wenn die Nase gereizt wird, und daß sie nicht selten zu gleicher Zeit rot werden. Den guten Ton wird sie ebenfalls nicht verlieren, wenn die Dienstfertige nicht zu sehr geklemmt

32 Der unwissende Mann ist natürlich B.Martin, «An essay on visual glasses».

${ }^{33}$ Diese Nachbilder hat auch Adams schon beschrieben. 
wird, und etwas Unterstützung durch Bügel an den Schläfen erhält und, was hier wohl bemerkt zu werden verdient, so hat es Leute gegeben, die diese im Dienst veränderte Sprache für schön gehalten haben, zumal wenn sie sich nicht sowohl dem näselnden Klarinettenton, als vielmehr der vornehmen halb erstickten Schnupftabakssprache nähert, die das $m$ fast wie $b$ ausspricht. Doch genug mit dieser Art zu widerlegen und von solchen Argumenten. - Die Lesegläser sind schädlich und unnütz: 1. weil sie ihrer Natur nach nicht festgehalten werden können, und also folglich das Auge immer andere Stellungen erfordert und auch annimmt, wodurch es ermüdet und geschwächt wird, daher solche Personen öfters sich genötigt sehen, zu Brillen überzugehen, wenn es fast zu spät ist; 2. weil das von ihrer Oberfläche zurückgeworfene Licht bei mancher Gelegenheit stark blendet und verwirrt und dadurch das Übel vermehrt; und 3. weil sie beim Schreiben und vielen andern Verrichtungen nicht zu gebrauchen sind. Personen, die in ihren besten Jahren kurzsichtig gewesen sind, bedürfen im Alter der Brillen selten oder gar nicht, weil ihr Auge zu viel Konvexität hatte, die sich nun verliert, aber nicht immer zu dem Grade, daß sie konvexer Brillen bedürften. Die Menge rechnet ihnen dieses zur Glückseligkeit, daß sie im späten Alter ohne Brillen lesen können, das heißt, nicht nötig haben, einen halben Gulden für ein paar Gläser hinzugeben, dafür sie dann die ganze übrige Lebenszeit für die Schönheit der Natur im großen blind waren, und nie den entzückenden Anblick einer schönen Gegend genossen haben. - Die Kurzsichtigen müssen sich bei der Wahl ihrer Brillen eben der Vorsicht bedienen, deren wir oben Erwähnung getan haben, nämlich ihre Gläser nicht gleich allzu hohl wählen, und würden wohl tun, sich bei Zeiten der Brillen von solcher Konkavität zu bedienen, die ihnen verstattet, das Buch acht bis zehn Zoll ${ }^{34}$ vom Auge zu halten, anstatt es dem bloßen Auge immer näherzubringen, und dadurch den Fehler immer mehr zu verschlimmern.

Noch muß ich denjenigen zum Trost erinnern, die von den kleinen schwarzen, vor den Augen schweben zu scheinenden Flecken geschreckt werden, welche die Franzosen "mouches volantes» nennen, daß sie wenig zu bedeuten haben. Ich kann hierin Herrn Prof. Büschs Erfahrung auch noch die meinige beifügen. Als ich mich im Jahre 1769 und 1770 sehr mit mikroskopischen Beobachtungen abgab, bemerkte ich ihrer mehrere, zumal im rechten Auge, nicht als wenn ich sie mir durch das Mikroskop zugezogen hätte, sondern weil die Lage des Auges, bei dem zusammengesetzten Mikroskop, da bei dem Abwärtssehen die Achse desselben fast vertikal zu stehen kommt, ihrer Beobachtung sehr günstig ist. Ich wurde dadurch beängstigt, zeichnete die Figur von einigen, um ihr Wachstum oder Abnahme zu bemerken, fing aber endlich an, mich nicht weiter mehr um sie zu kümmern, welches gegen viele Übel in der Welt, wo nicht ein treffliches Mittel selbst, doch gewiß eine große notwendige Unterstützung dabei ist, und fand nach fünf bis sechs Jahren unvermutet, daß die Flecken alle verschwunden waren. -

Außer den oben erwähnten Ursachen von Augenschwächen gibt es freilich auch noch andere, deren Hebung für den Arzt allein gehört. Aber die Schwäche entstehe, woher sie wolle, so wird allemal die oben erwähnte ökonomie beim Geschäfte des Sehens nötig sein, und jede Verabsäumung derselben die Sache verschlimmern.

$3420-23 \mathrm{~cm}$. 